\title{
ON RUIN PROBABILITY AND AGGREGATE CLAIM REPRESENTATIONS FOR PARETO CLAIM SIZE DISTRIBUTIONS
}

\author{
HAnsjörg Albrecher $^{a} \quad$ Dominik KortschaK ${ }^{a, b *}$ \\ a Institute of Actuarial Science, University of Lausanne, \\ Quartier UNIL-Dorigny, Batiment Extranef, CH-1015 Lausanne, Switzerland \\ ${ }^{b}$ Radon Institute for Computational and Applied Mathematics, Austrian Academy of Sciences, \\ Altenbergerstrasse 69, A-4040 Linz, Austria
}

\begin{abstract}
We generalize an integral representation for the ruin probability in a Crámer-Lundberg risk model with shifted (or also called US-)Pareto claim sizes, obtained by Ramsay [14], to classical Pareto(a) claim size distributions with arbitrary real values $a>1$ and derive its asymptotic expansion. Furthermore an integral representation for the tail of compound sums of Pareto-distributed claims is obtained and numerical illustrations of its performance in comparison to other aggregate claim approximations are provided.
\end{abstract}

\section{Introduction}

Consider the classical compound Poisson model of collective risk theory, where the surplus process $R(t)$ at time $t$ is given by

$$
R(t)=u+c t-\sum_{i=1}^{N(t)} X_{i}
$$

with $N(t)$ denoting a homogeneous Poisson process with intensity $\lambda$, the claim sizes $X_{i}$ are i.i.d. distributed non-negative random variables with distribution function $F$ and finite mean $\mu=\mathbb{E}\left(X_{i}\right), c$ is a constant premium intensity and the net profit condition $c>\lambda \mu$ holds. The ruin probability for a given initial surplus level $u$ is denoted by

$$
\psi(u)=\operatorname{Pr}[R(t)<0 \text { for some } t>0 \mid R(0)=u]
$$

and its properties are a classical object of study in risk theory (see for instance Asmussen [3]). In many situations it turns out that heavy-tailed distributions provide an appropriate fit to actual claim data and the focus of this paper will be on this case. Asymptotic properties of ruin probabilities $\psi(u)$ for large $u$ in the case of subexponential claim size distribution functions $F$ have been studied quite extensively in the literature (see for instance Teugels \& Veraverbeke [18], Embrechts \& Veraverbeke [9] and for higher-order asymptotic expansions Grübel [10], Omey \& Willekens [13], Willekens \& Teugels [21],

\footnotetext{
*Supported by the Austrian Science Foundation Project P18392-MAT and the Swiss National Science Foundation Project 200021-124635/1.
} 
Baltrūnas [5] and Barbe et al. [6]). On the other hand, apart from asymptotic results, also explicit expressions for $\psi(u)$ for heavy-tailed claim sizes are of interest, not the least for checking the accuracy of asymptotic estimates when applied to approximate the ruin probability $\psi(u)$ for moderate values of $u$. Among the heavy-tailed claim size distributions, the Pareto distribution is very popular in actuarial practice. For claims with so-called shifted (or US-)Pareto distribution function

$$
F(x)=1-\left(1+\frac{x}{m}\right)^{-m-1}, \quad x>0, m \in \mathbb{N},
$$

Ramsay [14] derived an interesting integral representation of $\psi(u)$, which only involves a single (non-oscillating) integral along the positive real line. In [15] this integral representation was generalized to arbritrary (non-integer) $m>0$, in [19] to a renewal risk model with Erlang interclaim times and in [16] to the case of compound shifted Pareto sums. In all these cases the used method is akin to the one used in [7] and [8] to determine the density function of the finite sum of certain Pareto variables.

It is natural to ask whether one can establish a similar formula for classical Pareto claims with density function

$$
f(x)=a d(d x)^{-(a+1)}, \quad x>\frac{1}{d}, a>1, d \geq 0,
$$

which is often preferred to the US-Pareto distribution by practitioners for modelling purposes.

In this paper, we will show that for Pareto distributions a slightly weaker result holds. In Section 2 a refined analysis of singularities in the complex domain is used to establish an integral representation of $\psi(u)$ for Pareto claim sizes with distribution function (2) and arbitrary positive parameter $a>1$. Two methods of proof are provided. Section 2.1 is an extension of the proof technique of Ramsay [14] to our situation. Section 2.2 gives a somewhat different proof which later on will allow us to extend the integral representation to other compound sums of Pareto random variables. In Section 3 we show how this new expression can be used to quickly obtain higher-order asymptotics of $\psi(u)$ for large $u$. While the ruin probability, by the Pollaczek-Khintchine formula (cf. [3, Chapter 3.2, p.61]), can be interpreted as a geometric compound of the integrated tail distribution, we indicate in Section 4 how to use the proof method of Section 2.2 to derive an integral representation for the tail of a compound sum of Pareto random variables. We especially highlight the case of compound Poisson sums and compound negative binomial sums of Pareto random variables (i.e. aggregate claims in the compound Poisson model or the compound negative binomial model, respectively). In Section 5 some numerical illustrations of the performance of the integral representations in comparison to other aggregate claim approximations are provided.

\section{An integral representation for the ruin probability with Pareto claims}

Since the parameter $d$ in (2) is just a scale parameter, we will without loss of generality assume $d=1$ in the sequel. 
Extending the approach of Ramsay [14] to the case of general Pareto claim sizes with density (2), we can derive an integral representation not for $\psi(s)$ itself, but for a function that has the same asymptotic expansion as $\psi(s)$. In Remark 2.4 we will indicate why strict equality between the integral representation and $\psi(s)$ can not hold in the case of general Pareto claims.

Define the Laplace transform of a function $g$ by

$$
\hat{L}_{g}(s)=\int_{0}^{\infty} e^{-s t} g(t) \mathrm{d} t
$$

From the usual integro-differential equation for the ruin probability in the Cramér-Lundberg model, it is not difficult to see that

$$
\hat{L}_{\psi}(s)=\frac{1}{s}-\frac{c-\lambda \mu}{c s-\lambda\left(1-\hat{L}_{f}(s)\right)}
$$

(see e.g. Rolski et al. [17, Equation (5.3.14), p.165]). Further note that for the Pareto density $f(x)=a x^{-a-1}$

$$
\hat{L}_{f}(s)=a s^{a} \Gamma(-a, s),
$$

where $\Gamma(a, s)=\int_{s}^{\infty} e^{-x} x^{a-1} \mathrm{~d} x$ is the incomplete Gamma function (see e.g. [1, Chapter 6.5, Page 260]) . For the ruin probability $\psi(u)$ in the compound Poisson model we then get

Theorem 2.1. Consider a compound Poisson model with Pareto claim size density $f(x)=$ $a x^{-a-1}$. If $\rho:=\lambda \mu / c=\lambda a /((a-1) c)$ and $\bar{F}_{I}(x)=1 / \mu \int_{x}^{\infty} \bar{F}(t) \mathrm{d} t$, then we have

$$
\begin{aligned}
& \psi(u) \approx \frac{\rho}{1-\rho} \bar{F}_{I}(u) \frac{(c-\lambda \mu)^{2}}{\Gamma(a-1)} \\
& \quad \times \int_{0}^{\infty} \frac{x^{a-2} e^{-x}}{\left(c+\lambda u / x \operatorname{Re}\left(1-\hat{L}_{f}\left(-\frac{x}{u}\right)\right)\right)^{2}+\left(\frac{\pi \lambda(x / u)^{a-1}}{\Gamma(a)}\right)^{2}} \mathrm{~d} x .
\end{aligned}
$$

where the notation $a(x) \approx b(x)$ means that there exists a $\delta>0$ with $|a(x)-b(x)|=\mathcal{O}\left(e^{-\delta x}\right)$ as $x \rightarrow \infty$.

Remark 2.1. Recall that $\lim _{u \rightarrow \infty} \psi(u) /\left(\frac{\rho}{1-\rho} \bar{F}_{I}(u)\right)=1$ (see e.g. [3, Theorem 2.1,p.259]).

Remark 2.2. The integrand in (5) is positive non-oscillating and of exponential decay. As in [14] the integral in (5) can be interpreted as the expected value of some function with respect to a Gamma distribution.

Remark 2.3. Note that all common asymptotic expansions of $\psi(u)$ (see e.g. [6]) have an error term that asymptotically is of the order $x^{-k}$ for a $k>0$, which is asymptotically worse than the exponential convergence rate of the error of the approximation (5). In that sense approximation (5) is more accurate than any asymptotic expansion (for a numerical illustration, cf. Section 5). 
Remark 2.4. From Theorem 2.1 we get for some integrable function $g(x)$

$$
\psi(u) \approx \int_{0}^{\infty} e^{-u x} g(x) \mathrm{d} x .
$$

A heuristic argument why we do not have equality in (6) is as follows: Due to the support of $F$ starting in $x=1, F_{I}^{* n}(x)$ is not differentiable at $x=n$, hence by the PollaczekKhintchine formula $\psi(u)$ is not holomorphic in the points $u=n$. On the other hand the integral in (6) is a Laplace transform and hence holomorphic for $\operatorname{Re}(u)>0$ so that we can not have equality, whereas in the US-Pareto case in [14], the support of F was the entire postive half-line and consequently $F_{I}^{* n}(x)$ was holomorphic in the entire right half plane and equality in (6) was feasible.

\subsection{Proof of Theorem 2.1, Method 1}

Proof of Theorem 2.1. A standard inversion formula of (3) yields

$$
\psi(u)=\frac{1}{2 \pi \iota} \int_{s_{0}-\iota \infty}^{s_{0}+\iota \infty} e^{u s} \hat{L}_{\psi}(s) \mathrm{d} s=1-\frac{1}{2 \pi \iota} \int_{s_{0}-\iota \infty}^{s_{0}+\iota \infty} e^{u s} \frac{c-\lambda \mu}{s c-\lambda\left(1-\hat{L}_{f}(s)\right)} \mathrm{d} s
$$

for an $s_{0}>0$, where $\iota=\sqrt{-1}$. From the definition of $\hat{L}_{f}(s)$ it follows that the integrand

$$
e^{u s} \frac{c-\lambda \mu}{s c-\lambda\left(1-\hat{L}_{f}(s)\right)}
$$

in (7) as a function of $s \in \mathbb{C}$ is meromorphic in the sliced plane $\mathbb{D}=\mathbb{C} \backslash(-\infty, 0]$. Hence, for the evaluation of (7) we can use a complex contour in $\mathbb{D}$, taking care of its enclosed poles, which are located at the zeroes of

$$
N(s):=c-\frac{\lambda}{s}\left(1-\hat{L}_{f}(s)\right) .
$$

Note that there will not be any pole inside the contour with positive real part because $\hat{L}_{\psi}(s)$ is bounded on that area except possibly for a branch point at $s=0$. Since $N(\bar{s})=\overline{N(s)}$ where $\bar{s}$ denotes the complex conjugate of $s$, we will concentrate our analysis on $\operatorname{Im}(s) \geq 0$. We will use the contour given in Figure 1 and we have to make sure that no zero of $N(s)$ lies on this contour. We will now state a series of lemmata, the proofs of which are postponed to the Appendix. The first two lemmata bound $|N(s)|$ from below. Lemma 2.2 establishes that for every $\pi / 2<\phi_{0}<\pi$ there exists an $r_{0}$ such that $1 / N(s)$ is bounded on the set $\left\{s=r e^{\iota \phi}: r>r_{0}\right.$ and $\left.\phi_{0}<\phi \leq \pi\right\}$ :

Lemma 2.2. For all $\epsilon>0$ and $\pi / 2<\phi_{0}<\pi$ there exists an $r_{0}$ such that for all $r>r_{0}$ and $\phi_{0} \leq \phi \leq \pi$

$$
\mid N(r(\cos (\phi)+\iota \sin (\phi)) \mid>\epsilon .
$$

The next lemma gives us a family of horizontal lines on which $1 / N(s)$ can be uniformly bounded and hence can be part of our contour.

Lemma 2.3. For all $\epsilon>0$, there exists a $k_{0}>0$ such that for all even integers $k>k_{0}$ and all $s:=-\alpha+\iota \beta(\alpha, \beta \in \mathbb{R})$ with $\alpha>0$ and $\beta=(2 k+1) \pi / 2$

$$
|N(s)|>c-\epsilon
$$




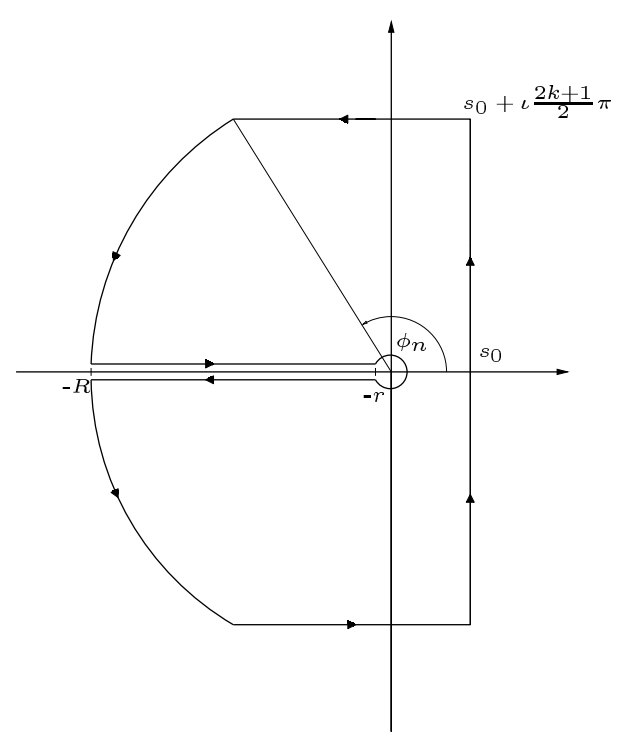

Figure 1: The contour integral

To evaluate the integral in (7) we now use the contour given in Figure 1. At first we choose a sequence $\phi_{n}$ with $3 \pi / 2<\phi_{n}<\pi / 2$ and $\lim _{n \rightarrow \infty} \phi_{n}=\pi / 2$. Then we choose an even integer $k_{n}$ such that for $|s| \geq\left(2 k_{n}+1\right) \pi / 2$ and for $\phi_{n} \leq \arg (s) \leq \pi, N(s)>\delta_{0}$ (this can be done because of Lemma 2.2). Further we assume that $k_{n}>k_{0}$ as defined in Lemma 2.3 (where we choose $\left.\epsilon<c-\delta_{0}\right)$ and define $R_{n}=\left(2 k_{n}+1\right) \pi /\left(2 \sin \left(\phi_{n}\right)\right)$. The contour consists of the line $s_{0}+\iota x$ for $x \in\left[-\left(2 k_{n}+1\right) \pi / 2,\left(2 k_{n}+1\right) \pi / 2\right]$, the lines $x \pm \iota\left(2 k_{n}+1\right) \pi / 2$ for $x \in\left[R_{n} \cos \left(\phi_{n}\right), s_{0}\right]$, the semi-circle $C_{R_{n}}=\left\{z:|z|=R_{n}, \phi_{n} \leq \arg (z) \leq 2 \pi-\phi_{n}\right\}$, the line from $\left[-R_{n},-r\right]$ lying above the branch cut of the negative real axis with $r<R_{n}$, the circle $C_{r}=\{z:|z|=r, z \neq-r\}$ and the line from $\left[-r,-R_{n}\right]$ lying below the branch cut of the negative real axis (see Figure 1). From the residue theorem one obtains

$$
\begin{aligned}
& -\frac{1}{2 \pi \iota} \int_{s_{0}-\iota R}^{s_{0}+\iota R} e^{u s} \frac{c-\lambda \mu}{c s-\lambda\left(1-\hat{L}_{f}(s)\right)} \mathrm{d} s \\
= & \frac{1}{2 \pi \iota}\left(\int_{C_{R_{n}}}+\int_{s_{0}+\iota \frac{\left(2 k_{n}+1\right) \pi}{2}}^{R_{n} \cos \left(\phi_{n}\right)+\iota \frac{\left(2 k_{n}+1\right) \pi}{2}}+\int_{R_{n} \cos \left(\phi_{n}\right)-\iota \frac{\left(2 k_{n}+1\right) \pi}{2}}^{s_{0}-\iota \frac{\left(2 k_{n}+1\right) \pi}{2}}+\int_{-R}^{-r}+\int_{C_{r}}^{2}+\int_{-r}^{-R}\right) e^{u s} \frac{c-\lambda \mu}{c s-\lambda\left(1-\hat{L}_{f}(s)\right)} \mathrm{d} s \\
& -\sum_{i=1}^{M_{n}} \operatorname{Res}_{s_{i}}\left(e^{u s} \frac{c-\lambda \mu}{c s-\lambda\left(1-\hat{L}_{f}(s)\right)}\right)
\end{aligned}
$$

where $s_{i}, i=1, \ldots, M_{n}$ are the zeros of $N(s)$ that lie inside the contour.

By using Lemma 2.2 and the asymptotic behavior of the Gamma function, it is straightforward to see that

$$
\lim _{n \rightarrow \infty} \frac{1}{2 \pi \iota} \int_{C_{R_{n}}} \frac{1}{s} e^{u s} \frac{c-\lambda \mu}{c-\frac{\lambda}{s}\left(1-\hat{L}_{f}(s)\right)} \mathrm{d} s=0 .
$$


Furthermore,

$$
\lim _{r \rightarrow 0}=\frac{1}{2 \pi \iota} \int_{C_{r}} e^{u s} \frac{1}{s} \frac{c-\lambda \mu}{c-\frac{\lambda}{s}\left(1-\hat{L}_{f}(s)\right)} \mathrm{d} s=-1
$$

is a consequence of $\lim _{s \rightarrow 0}\left(1-\hat{L}_{f}(s)\right) / s=\mu$, cf. (15) below.

From Lemma 2.3 we get:

$$
\begin{array}{r}
\left|\int_{s_{0}+\iota \frac{\left(2 k_{n}+1\right) \pi}{2}}^{R_{n} \cos \left(\phi_{n}\right)+\iota \frac{\left(2 k_{n}+1\right) \pi}{2}} e^{u s} \frac{1}{s} \frac{c-\lambda \mu}{N(s)} \mathrm{d} s\right| \leq \frac{c-\lambda \mu}{\delta_{0}} \frac{2}{\left(2 k_{n}+1\right) \pi} \int_{R_{n} \cos \left(\phi_{n}\right)}^{s_{0}} e^{u s} \mathrm{~d} s \\
=\frac{c-\lambda \mu}{\delta_{0}} \frac{2}{\left(2 k_{n}+1\right) \pi}\left(e^{u s_{0}}-e^{u R_{n} \cos \left(\phi_{n}\right)}\right),
\end{array}
$$

which tends to 0 as $n \rightarrow \infty$. For $x \in \mathbb{R}^{-}$define $\hat{L}_{f}(x)=\lim _{y \rightarrow 0^{+}} \hat{L}_{f}(x+\iota y)$. With $\hat{L}_{f}(\bar{x})=\bar{L}_{f}(x)$, we get as in [14] that the sum of the two remaining integrals can be expressed as

$$
\frac{1}{\pi} \int_{r}^{R} e^{-u x} \frac{\lambda(c-\lambda \mu) \operatorname{Im} \hat{L}_{f}(-x)}{\left|c x+\lambda\left(1-\hat{L}_{f}(-x)\right)\right|^{2}} \mathrm{~d} x .
$$

Taking the limit $R \rightarrow \infty$ and $r \rightarrow 0$ yields

$$
\psi(u)=-\frac{1}{\pi} \int_{0}^{\infty} e^{-u x} \frac{\lambda(c-\lambda \mu) \operatorname{Im}\left(\hat{L}_{f}(-x)\right)}{\left|c x+\lambda\left(1-\hat{L}_{f}(-x)\right)\right|^{2}} \mathrm{~d} x-\sum_{i=1}^{\infty} \operatorname{Res}_{s_{i}}\left(e^{u s} \frac{c-\lambda \mu}{c s-\lambda\left(1-\hat{L}_{f}(s)\right)}\right)
$$

We have to show that

$$
\sum_{i=1}^{\infty} \operatorname{Res}_{s_{i}}\left(e^{u s} \frac{c-\lambda \mu}{c s-\lambda\left(1-\hat{L}_{f}(s)\right)}\right)=\mathcal{O}\left(e^{-\delta u}\right) .
$$

For a single $s_{i}$ it is quite obvious that for every $\epsilon>0$

$$
\operatorname{Res}_{s_{i}}\left(e^{u s} \frac{c-\lambda \mu}{c s-\lambda\left(1-\hat{L}_{f}(s)\right)}\right)=\mathcal{O}\left(e^{\left(\operatorname{Re}\left(s_{i}\right)+\epsilon\right) u}\right) .
$$

Hence we have to ensure that by summing over all $s_{i}$ this property remains to hold. For this we are only interested in $s_{i}$ with $\left|s_{i}\right|$ large. To determine the location of the zeros of $N(s)$ (and hence the $s_{i}$ needed to evaluate the residuals of the integrand), the following lemma gives an asymptotic relation between $\operatorname{Re}(s)$ and $\operatorname{Im}(s)$ of solutions of $N(s)=0$.

Lemma 2.4. For every $0<\epsilon<\min (c, 1)$, there exists a $\beta_{0}>0$ such that for each zero $s:=-\alpha+\iota \beta$ of $N(s)$ with $\beta>\beta_{0}$, its negative real part $\alpha>0$ satisfies

$$
\log \left(\frac{(c-\epsilon) \beta^{2}}{a \lambda(1+\epsilon)}\right) \leq \alpha \leq \log \left(\frac{(1+\epsilon)(c+\epsilon) \beta^{2}}{a \lambda(1-\epsilon)}\right) .
$$

The next lemma provides expressions for $\operatorname{Im}(s)$ for $N(s)=0$. Together with Lemma 2.4 we can find expressions for the zeros of $N(s)$. 
Lemma 2.5. For every $0<\epsilon<\min (c, 1)$ there exists a $\beta_{0}>0$ such that for each zero $s:=-\alpha+\iota \beta$ of $N(s)$ with $\beta>\beta_{0}$,

$$
|\sin \beta|<\epsilon \quad \text { and } \quad|\cos \beta-1|<\epsilon .
$$

After we approximately know where the zeros of $N(s)$ are located we have to make sure that there are not too many zeros close to each other in order to guarantee (10). Further we will show that there exist zeros of $N(s)$.

Lemma 2.6. There exists a constant $c_{0}$ such that for every $k>0$ the number of zeros of $N(s)$ inside the circle $K_{1}\left(s_{k}\right)$ around $s_{k}=-\log \left(c(2 k \pi)^{2} /(a \lambda)\right)+\iota 2 k \pi$ with radius 1 is less than $c_{0}$. Moreover, there exists a $k_{0}$ such that for every $k>k_{0}$ the number of zeros of $N(s)$ inside the circle $K_{1}\left(s_{k}\right)$ is 1 .

Next one can bound the order of the zeros of $N(x)$ which is needed to bound the residuals.

Lemma 2.7. $A s \in \mathbb{D}$ with $N(s)=N^{\prime}(s)=N^{\prime \prime}(s)=0$ and there exists an $M>0$ such that for $|s|>M, N(s)=N^{\prime}(s)=0$ has no solution.

The next lemma provides an exponential bound for a single residual at a pole $s_{i}$ of the integrand (8) with $\left|s_{i}\right|$ large.

Lemma 2.8. For every $\epsilon>0$ there exists a $\beta_{1}>0$ such that for $s_{i}=-\alpha+\iota \beta$ with $\beta>\beta_{0}$ and $N\left(s_{i}\right)=N(-\alpha+\iota \beta)=0$ and every $k>0$

$$
\begin{aligned}
\mid \operatorname{Res}_{s_{i}}\left(e^{u s} \frac{1}{s}\right. & \left.\frac{c-\lambda \mu}{c-\frac{\lambda}{s}\left(1-\hat{L}_{f}(s)\right)}\right) \mid \\
& \leq e^{-(u-k) \alpha} \frac{(c-\lambda \mu)\left(\frac{a \lambda(1+\epsilon)}{c-\epsilon}\right)^{k}\left(\alpha^{2}+\beta^{2}\right)}{a \lambda\left(\left(\frac{c-\epsilon}{a \lambda(1+\epsilon)}\right) \beta^{2}-\frac{\left(\alpha^{2}+\beta^{2}\right)^{1 / 2}}{\rho}-1\right)} \beta^{-2 k-1} \leq e^{-(u-k) \alpha} \beta^{-2 k+1} .
\end{aligned}
$$

Hence (10) is proven and the integral representation follows by algebraic manipulations of (9).

\subsection{Proof of Theorem 2.1, Method 2:}

Before we identify an alternative proof of Theorem 2.1, we will need an auxiliary result.

Lemma 2.9. Let $g(s)$ be any meromorphic function on $\mathbb{D} \cap\left\{s: \operatorname{Re}(s)>-t_{0}\right\}$ for a $t_{0}>0$, with a branch cut on the line $\left(-t_{0}, 0\right]$ (for $0<x<t_{0}$ define $g(-x):=\lim _{y \rightarrow 0+} g(-x+\iota y)$ ). If further $g(\bar{s})=\overline{g(s)}, g(s)$ has no pole for $\operatorname{Re}(s) \geq 0, \lim _{s \rightarrow 0} g(s)=1$, and if there exists $a c_{0}$ and an $\epsilon>0$ such that, uniformly for $\alpha$ in bounded intervals,

$$
\left|c_{0}-g(\alpha+\iota \beta)\right|=\mathcal{O}\left(\frac{1}{\beta^{\epsilon}}\right), \text { as } \beta \rightarrow \infty,
$$

then there exists a $\delta>0$ with

$$
\frac{1}{2 \pi \iota} \int_{s_{0}-\iota \infty}^{s_{0}+\iota \infty} e^{u s} \frac{1}{s} g(s) \mathrm{d} s=1-\frac{1}{\pi} \int_{0}^{t_{0}} e^{-u x} \frac{1}{x} \operatorname{Im}(g(-x)) \mathrm{d} x+\mathcal{O}\left(e^{-\delta u}\right) .
$$




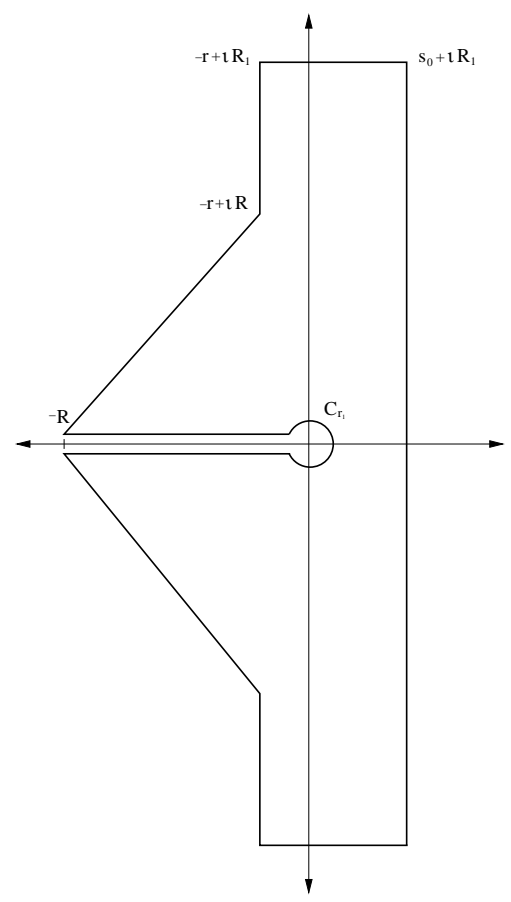

Figure 2: The contour integral

If further $g(-x)$ can be defined for $x \in\left(-\infty, t_{0}\right]$ and there exists a $\delta_{0}>0$ with

$$
\int_{0}^{\infty}\left|e^{-\delta_{0} x} \frac{1}{x} \operatorname{Im}(g(-x))\right| \mathrm{d} x<\infty
$$

then there exists a $\delta>0$ with

$$
\frac{1}{2 \pi \iota} \int_{s_{0}-\iota \infty}^{s_{0}+\iota \infty} e^{u s} \frac{1}{s} g(s) \mathrm{d} s=1-\frac{1}{\pi} \int_{0}^{\infty} e^{-u x} \frac{1}{x} \operatorname{Im}(g(-x)) \mathrm{d} x+\mathcal{O}\left(e^{-\delta u}\right) .
$$

Proof. At first choose an $0<r<t_{0}$ and $0<R<t_{0}$ such that for a $c_{1}<\infty$, for all $\alpha \in\left[-r, s_{0}\right]$ and all $\beta>R$

$$
\left|c_{0}-g(\alpha+\iota \beta)\right| \leq \frac{c_{1}}{\beta^{\epsilon}} .
$$

For $R_{1}>0, r_{1}>0$ we will use the contour given in Figure 2 consisting of the line $s_{0}+\iota x$, $x \in\left[-R_{1}, R_{1}\right]$, the lines $x \pm \iota\left(R_{1}\right)$ for $x \in\left[-r, s_{0}\right]$, the lines $-r \pm x$ for $x \in\left[R, R_{1}\right]$, the complex paths $\gamma_{ \pm}(t)$ for $t \in[0,1]$ connecting the points $\{-r \pm \iota R,-R\}$ with $-t_{0}<$ $\operatorname{Re}\left(\gamma_{ \pm}(t)\right) \leq-r$, where $\gamma_{ \pm}(t)$ does not cross any poles of $g(s)$ and the length $L\left(\gamma_{ \pm}\right)$is bounded, the line from $\left[-R,-r_{1}\right]$ lying above the branch cut of the negative real axis, the circle $C_{r_{1}}=\left\{s:|s|=r_{1}, s \neq-r_{1}\right\}$ and the line from $\left[-r_{1},-R\right]$ lying below the branch cut of the negative real axis. Note that

$$
\left|\int_{s_{0} \pm \iota R_{1}}^{-r \pm \iota R_{1}} e^{u s} \frac{1}{s} g(s) \mathrm{d} s\right| \leq\left(s_{0}+r\right) e^{s_{0} u} \frac{1}{R_{1}}\left(1+\frac{c_{1}}{R_{1}^{\epsilon}}\right)
$$


which tends to 0 as $R_{1} \rightarrow \infty$. Further, from $\lim _{s \rightarrow 1} e^{u s} g(s)=1$ it follows that

$$
\lim _{r_{1} \rightarrow 0} \int_{C_{r_{1}}} e^{u s} \frac{1}{s} g(s) \mathrm{d} s=-1 .
$$

From $g(\bar{s})=\overline{g(s)}$ we have

$$
\begin{aligned}
\frac{1}{2 \pi \iota}\left(\int_{-R}^{-r_{1}} e^{u x} \frac{1}{x} g(x+\iota 0) \mathrm{d} x+\int_{-r_{1}}^{-R} e^{-u x} \frac{1}{x} g(x-\iota 0) \mathrm{d} x\right) \\
=\frac{1}{2 \pi \iota} \int_{-R}^{-r_{1}} e^{u x} \frac{1}{x} g(x)-e^{-u x} \frac{1}{x} \overline{g(x)} \mathrm{d} x=\frac{1}{\pi} \int_{r_{1}}^{R} e^{-u x} \frac{1}{x} \operatorname{Im}(g(-x)) \mathrm{d} x .
\end{aligned}
$$

By the Cauchy Residual Theorem it follows that

$$
\begin{aligned}
& \frac{1}{2 \pi \iota} \int_{s_{0}-\iota \infty}^{s_{0}+\iota \infty} e^{u s} \frac{1}{s} g(s) \mathrm{d} s-\left(1-\frac{1}{\pi} \int_{0}^{R} e^{-u x} \frac{1}{x} \operatorname{Im}(g(-x)) \mathrm{d} x\right) \\
& \quad=-\frac{1}{2 \pi \iota}\left(\int_{\gamma_{+}}+\int_{\gamma_{-}}+\int_{-r+\iota \infty}^{-r+\iota R}+\int_{-r-\iota R}^{-r-\iota \infty}\right) e^{u s} \frac{1}{s} g(s) \mathrm{d} s+\sum_{i=1}^{M_{R, r}} \operatorname{Res}_{s_{i}}\left(e^{u s} \frac{1}{s} g(s)\right)
\end{aligned}
$$

where $s_{i}, i=1, \ldots, M_{R, r}$ are the poles of $g(s)$ inside the contour. Since $g(s)$ has no poles with $\operatorname{Re}(s) \geq 0$, there exists a $\delta_{1}>0$ with $-\delta_{1}>\operatorname{Re}\left(s_{i}\right)$ for all $i=1, \ldots, M_{R, r}$ and hence

$$
\operatorname{Res}_{s_{i}}\left(e^{u s} \frac{1}{s} g(s)\right)=\mathcal{O}\left(e^{-\delta u}\right) .
$$

We have

$$
\left|\int_{\gamma_{ \pm}} e^{u s} \frac{1}{s} g(s)\right| \leq L\left(\gamma_{ \pm}\right) \max _{t \in[0,1]}\left|e^{u \gamma_{ \pm}(t)} \frac{1}{\gamma_{ \pm}(t)} g\left(\gamma_{ \pm}(t)\right)\right| \leq e^{-r u} L\left(\gamma_{ \pm}\right) \max _{t \in[0,1]}\left|\frac{1}{\gamma_{ \pm}(t)} g\left(\gamma_{ \pm}(t)\right)\right| \text {. }
$$

Further

$$
\int_{-r+\iota R}^{-r+\iota \infty} e^{u s} \frac{1}{s} g(s) \mathrm{d} s=c_{0} \int_{-r+\iota R}^{-r+\iota \infty} e^{u s} \frac{1}{s} \mathrm{~d} s+\int_{-r+\iota R}^{-r+\iota \infty} e^{u s} \frac{1}{s}\left(g(s)-c_{0}\right) \mathrm{d} s .
$$

Note that

$$
\begin{aligned}
\left|\int_{-r+\iota R}^{-r+\iota \infty} e^{u s} \frac{1}{s}\left(g(s)-c_{0}\right) \mathrm{d} s\right| \leq e^{-r u} \int_{R}^{\infty}\left|\frac{1}{-r+\iota x}\left(g(-r+\iota x)-c_{0}\right)\right| \mathrm{d} s & \\
& \leq e^{-r u} \int_{R}^{\infty} \frac{c_{1}}{x^{1+\epsilon}} \mathrm{d} x=\frac{c_{1}}{\epsilon R^{\epsilon}} e^{-r u} .
\end{aligned}
$$

Since there exists a $c_{2}$ such that for all $u>u_{0}$

$$
\left|\int_{R}^{\infty} e^{\iota u x} \frac{1}{-r+\iota x} \mathrm{~d} x\right| \leq c_{2}
$$

we have

$$
\left|\int_{-r+\iota R}^{-r+\iota \infty} e^{u s} \frac{1}{s} \mathrm{~d} s\right| \leq c_{2} e^{-u r} .
$$


The assertion finally follows by $\left(u>\delta_{0}\right)$ :

$$
\left|\int_{R}^{\infty} e^{-u x} \frac{1}{x} \operatorname{Im}(g(-x)) \mathrm{d} x\right| \leq e^{-R\left(u-\delta_{0}\right)} \int_{R}^{\infty} \frac{1}{x}\left|e^{-\delta_{0} x} \operatorname{Im}(g(-x))\right| \mathrm{d} x,
$$

where $g(-x)$ is interpreted as 0 where it is not defined.

Proof of Theorem 2.1. From (7) we see that we have to apply Lemma 2.9 with

$$
g(s)=\frac{c-\lambda \mu}{c-\frac{\lambda}{s}\left(1-\hat{L}_{f}(s)\right)} .
$$

Clearly $g(\bar{s})=\overline{g(s)}$. For $\operatorname{Re}(s) \geq 0$ we get

$$
\begin{aligned}
\left|1-\hat{L}_{f}(s)\right| & =\left|1-\int_{1}^{\infty} e^{-s x} a t^{-a-1} \mathrm{~d} x\right|=\left|1-e^{-s}+s \int_{1}^{\infty} e^{-s x} x^{-a} \mathrm{~d} x\right| \\
& =\left|s \int_{0}^{\infty} e^{-s x} \bar{F}(x) \mathrm{d} x\right| \leq\left|s \int_{0}^{\infty} \bar{F}(x) \mathrm{d} x\right|=|s| \mu,
\end{aligned}
$$

so that $g(s)$ has no poles with $\operatorname{Re}(s) \geq 0$, due to the net profit condition $c>\lambda \mu$ (alternatively, one could also argue except for a possible branch point at $s=0, \hat{L}_{\psi}(s)$ has no poles with $\operatorname{Re}(s) \geq 0)$.

An asymptotic expansion of $\Gamma(-a, s)$ (cf. [12, Chapter 4]) gives

$$
\Gamma(-a, s)=s^{-a-1} e^{-s}\left(1+\sum_{i=1}^{n-1} \frac{\prod_{j=1}^{i}(-a-j)}{s^{-i}}+\epsilon_{n}(s)\right),
$$

where $\left|s^{n} \epsilon_{n}(s)\right|$ can be uniformly bounded for large $|s|$. It follows that $\hat{L}_{f}(s) \sim \frac{a}{s} e^{-s}$, from which we get that, uniformly for $-r \leq \operatorname{Re}(s) \leq s_{0}\left(r, s_{0}>0\right), 1-\hat{L}_{f}(s) \rightarrow 1$ as $|s| \rightarrow \infty$. Hence

$$
\left|\frac{c-\lambda \mu}{c}-g(\alpha+\iota \beta)\right|=\mathcal{O}\left(\frac{1}{\beta}\right) \text {. }
$$

From [1, Equation 6.5.29, Page 262] we get that for $a \notin \mathbb{N}$

$$
\hat{L}_{f}(s)=a s^{a} \Gamma(-a)-a \Gamma(-a) \gamma^{*}(-a, s)=a s^{a} \Gamma(-a)-a \sum_{n=0}^{\infty} \frac{(-s)^{n}}{(n-a) n !} .
$$

On the other hand, for $a \in \mathbb{N}$ define

$$
E_{a}(x)=\int_{1}^{\infty} \frac{e^{-x t}}{t^{a}} \mathrm{~d} t
$$

leading to (cf. [1, Equation 5.1.12, Page 229])

$$
\hat{L}_{f}(s)=a E_{a+1}(s)=\frac{(-s)^{a}}{(a-1) !}\left(-\log (s)-\gamma+\sum_{n=1}^{a} \frac{1}{n}\right)-a \sum_{\substack{n=0 \\ n \neq a}}^{\infty} \frac{(-s)^{n}}{(n-a) n !},
$$


where $\gamma=0.577216 \ldots$ is Euler's constant. In both cases it follows

$$
\lim _{s \rightarrow 0} \frac{1}{s}\left(1-\hat{L}_{f}(s)\right)=\frac{a}{a-1}=\mu, \quad \text { or equivalently } \quad \lim _{s \rightarrow 0} g(s)=1 .
$$

Note that

$$
\operatorname{Im}(g(-x))=\frac{\frac{\lambda}{x} \operatorname{Im}\left(\hat{L}_{f}(-x)\right)}{\left(c+\lambda / x \operatorname{Re}\left(1-\hat{L}_{f}(-x)\right)\right)^{2}+\frac{\lambda^{2}}{x^{2}} \operatorname{Im}\left(\hat{L}_{f}(-x)\right)^{2}}
$$

and (cf. (15), (16))

$$
\operatorname{Im}\left(\hat{L}_{f}(-x)\right)=-\frac{\pi x^{a}}{\Gamma(a)},
$$

so that for every $\delta_{0}>0(12)$ holds. The theorem finally follows with Lemma 2.9 and some algebraic manipulations.

\section{Asymptotic expansion of the ruin probability}

Expansions for ruin probabilities in the Cramér-Lundberg model can be derived from expansions for compound distributions whenever the ladder height distribution of the claim size distribution

$$
F_{I}(u)=\frac{1}{\mu} \int_{0}^{u} \bar{F}(x) d x
$$

admits expansions for convolutions, see e.g [6] or [20]. For instance, if the claim size distribution $F(u)$ has a density $f(u)$ such that its negative derivative $-f^{\prime}(u)$ is regularly varying with index $-a-2, a>3$ (such that the moments $\mu_{k}:=a /(a-k)=\mathbb{E}\left[X^{k}\right]$ are finite for $k=1,2,3)$, then the expansion for the infinite horizon ruin probability $\psi(u)$ of third order reads (cf. [20])

$$
\psi(u)=\frac{\rho}{1-\rho} \bar{F}_{I}(u)+\frac{\rho^{2}}{(1-\rho)^{2}} \frac{\mu_{2}}{2 \mu^{2}} \bar{F}(u)+\left(\frac{\rho^{2}}{(1-\rho)^{2}} \frac{\mu_{3}}{3 \mu^{2}}+\frac{\rho^{3}}{(1-\rho)^{3}} \frac{\mu_{2}^{2}}{4 \mu^{3}}\right) f(s)+o(f(s)) .
$$

In this section we are going to show that an asymptotic expansion of (5) indeed retains (18). An advantage of this alternative method to derive expansions is that one can also derive the asymptotic expansion for values $a<3$. In particular, we will see that for every noninteger $a>2$ the expansion

$$
\begin{aligned}
\psi(u)=\frac{\rho}{1-\rho} \bar{F}_{I}(u) & +\frac{\rho^{2}}{(1-\rho)^{2}} \frac{\mu_{2}}{\mu^{2}} \bar{F}(u)+\left(\frac{\rho^{3}}{(1-\rho)^{3}} \frac{3 \mu_{2}^{2}}{4 \mu^{3}}+\frac{\rho^{2}}{(1-\rho)^{2}} \frac{\mu_{3}}{3 \mu^{2}}\right) f(u) \\
- & \frac{\pi}{\tan (a \pi)} \frac{\rho^{2}}{(1-\rho)^{2}} \frac{2 \Gamma(2(a-1))}{\Gamma(a) \Gamma(a-1)} \bar{F}_{I}(u)^{2}+\mathcal{O}\left(u^{-\min \{a+2,2 a-1\}}\right),
\end{aligned}
$$

where for $a<3$ the first term in the second line of (19) will dominate the $\mathcal{O}$-term.

Remark 3.1. In (19), one observes that for $a=(2 n+1) / 2(n \in \mathbb{N})$, the constant in front of $\bar{F}_{I}(u)^{2}$ in the expansion vanishes, which illustrates the special role played by these values of the shape parameter $a$. 
From now on we will assume that $a \notin \mathbb{N}$. At first we want to look for an expansion of the denominator of the integral (5). Define

$$
c_{1}=\frac{\pi}{\Gamma(a) \tan (a \pi)} \quad \text { and } \quad a_{n}=\frac{a}{(n+1-a)(n+1) !},
$$

to get

$$
\frac{1-\operatorname{Re}\left(\hat{L}_{f}(-x)\right)}{x}=c_{1} x^{a-1}+\sum_{n=0}^{\infty} a_{n} x^{n} .
$$

With

$$
c_{2}=-\frac{\pi}{\Gamma(a)} \quad \text { and } \quad b_{n}=\sum_{k=0}^{n} a_{k} a_{n-k}
$$

we obtain

$$
\begin{aligned}
& \left(c+\lambda / x\left(1-\operatorname{Re}\left(\hat{L}_{f}(-x)\right)\right)\right)^{2}+\frac{\lambda^{2}}{x^{2}} \operatorname{Im}\left(\hat{L}_{f}(-x)\right)^{2} \\
& =c^{2}+2 c \lambda \frac{1-\operatorname{Re}\left(\hat{L}_{f}(-x)\right)}{x}+\lambda^{2}\left(\frac{1-\operatorname{Re}\left(\hat{L}_{f}(-x)\right)}{x}\right)^{2}+\frac{\lambda^{2}}{x^{2}} \operatorname{Im}\left(\hat{L}_{\bar{F}}(-x)\right)^{2} \\
& =c^{2}+\lambda^{2}\left(c_{1}^{2}+c_{2}^{2}\right) x^{2 a-2}+2 \lambda c\left(c_{1} x^{a-1}+\sum_{n=0}^{\infty} a_{n} x^{n}\right) \\
& +2 \lambda^{2} c_{1} x^{a-1} \sum_{n=0}^{\infty} a_{n} x^{n}+\lambda^{2} \sum_{n=0}^{\infty} b_{n} x^{n} .
\end{aligned}
$$

Define

$$
\begin{aligned}
c_{0} & :=\lambda^{2}\left(c_{1}^{2}+c_{2}^{2}\right), \\
d_{n} & := \begin{cases}2 \lambda^{2} c_{1} a_{0}+2 \lambda c c_{1} & n=0, \\
2 \lambda^{2} c_{1} a_{n} & n \geq 1,\end{cases} \\
e_{n} & := \begin{cases}c^{2}+\lambda^{2} b_{0}+2 \lambda c a_{0} & n=0, \\
\lambda^{2} b_{n}+2 \lambda c a_{n} & n \geq 1 .\end{cases}
\end{aligned}
$$

Note that if $a=(2 k+1) / 2$ for $k \in \mathbb{N}$, then $c_{1}=0$. We get

$$
\begin{aligned}
\left(c+\lambda / x\left(1-\operatorname{Re}\left(\hat{L}_{f}(-x)\right)\right)\right)^{2}+\frac{\lambda^{2}}{x^{2}} \operatorname{Im}\left(\hat{L}_{f}(\right. & -x))^{2} \\
& =c_{0} x^{2 a-2}+x^{a-1} \sum_{n=0}^{\infty} d_{n} x^{n}+\sum_{n=0}^{\infty} e_{n} x^{n} .
\end{aligned}
$$

For ease of notation let

$$
N_{u}(x):=\left(c+\frac{u \lambda}{x}\left(1-\operatorname{Re}\left(\hat{L}_{f}\left(-\frac{x}{u}\right)\right)\right)\right)^{2}+\frac{u^{2} \lambda^{2}}{x^{2}} \operatorname{Im}\left(\hat{L}_{f}\left(-\frac{x}{u}\right)\right)^{2},
$$


and

$$
Z_{u}\left(x, n_{0}, n_{1}, n_{2}\right):=I_{\left\{n_{2}=0\right\}} c_{0}\left(\frac{x}{u}\right)^{2 a-2}+\left(\frac{x}{u}\right)^{a-1} \sum_{n=n_{1}}^{\infty} d_{n}\left(\frac{x}{u}\right)^{n}+\sum_{n=n_{0}}^{\infty} e_{n}\left(\frac{x}{u}\right)^{n} .
$$

Note that

$$
N_{u}(x)=Z_{u}(x, 0,0,0) .
$$

To find an asymptotic expansion for (5) we first give an expansion for integrals of the form

$$
\int_{0}^{\infty} \frac{Z_{u}\left(x, n_{0}, n_{1}, n_{2}\right) x^{m(a-1)+n-1} e^{-x}}{N_{u}(x)} \mathrm{d} x,
$$

where $n_{0}, m \geq 1$ and $n_{1}, n_{2}, n, k \geq 0$.

Lemma 3.1. We have

$$
\begin{aligned}
\int_{0}^{\infty} & \frac{Z_{u}\left(x, n_{0}, n_{1}, n_{2}\right) x^{m(a-1)+n-1} e^{-x}}{N_{u}(x)} \mathrm{d} x \\
= & I_{\left\{n_{2}=0\right\}} c_{0} u^{-2(a-1)} \int_{0}^{\infty} \frac{x^{(m+2)(a-1)+n-1} e^{-x}}{N_{u}(x)} \mathrm{d} x \\
& +\sum_{i=n_{1}}^{\infty} d_{i} u^{-(a-1)-i} \int_{0}^{\infty} \frac{x^{(m+1)(a-1)+n+i-1} e^{-x}}{N_{u}(x)} \mathrm{d} x \\
& +\sum_{i=n_{0}}^{\infty} e_{i} u^{-i} \int_{0}^{\infty} \frac{x^{m(a-1)+n+i-1} e^{-x}}{N_{u}(x)} \mathrm{d} x .
\end{aligned}
$$

Proof. There exists an $M>0$ such that $M \geq 1 / N_{u}(x)$ for all $x \geq 0$. We get for $\beta>0$ and $i \geq 0$

$$
\int_{0}^{\infty} \frac{x^{\beta-1+i} e^{-x}}{N_{u}(x)} \mathrm{d} x \leq M \int_{0}^{\infty} x^{\beta-1+i} e^{-x} \mathrm{~d} x=M \Gamma(\beta+i) .
$$

It then suffices to show that

$$
\sum_{i=n_{1}}^{\infty} d_{i} u^{-i} \Gamma(\beta+i) \quad \text { and } \quad \sum_{i=n_{0}}^{\infty} e_{i} u^{-i} \Gamma(\beta+i),
$$

are absolutely convergent series. We have for $i>1$

$$
d_{i} \Gamma(\beta+i)=2 \lambda^{2} c_{1} \frac{a \Gamma(\beta+i)}{(i+1-a) \Gamma(i+2)} .
$$

Since for $\alpha>0, m \in \mathbb{N}, m+1+b>\alpha>m+b,-b \notin \mathbb{N}$, and $i>m+1$

$$
\begin{aligned}
& \frac{\Gamma(\alpha+i)}{\Gamma(b+i)}=\frac{\Gamma(\alpha) \prod_{j=0}^{i-1}(\alpha+j)}{\Gamma(b) \prod_{j=0}^{i-1}(b+j)} \\
&=\frac{\Gamma(\alpha)}{\Gamma(b) \prod_{j=0}^{m}(b+j)} \prod_{j=0}^{i-m-2} \frac{\alpha+j}{b+m+1+j} \prod_{j=0}^{m}(\alpha-j-1+i),
\end{aligned}
$$


we obtain that

$$
\sum_{i=n_{1}}^{\infty} d_{i} u^{-i} \Gamma(\beta+i)
$$

converges absolutely for all $u>1$. For the second sum, note that for $n>1, e_{n}=$ $\lambda^{2} b_{n}+2 \lambda a_{n}$, hence we have to show that $\sum_{i=n_{0}} b_{i} \Gamma(\beta+i) u^{-i}$ is absolutely convergent. But for $u>2$ the latter follows from

$$
\begin{aligned}
\left|b_{n}\right| & =\left|\sum_{k=0}^{n} \frac{a^{2}}{(k+1-a)(k+1) !(n-k+1-a)(n-k+1) !}\right| \\
& \leq \frac{a^{2}}{\left(\min _{z \in \mathbb{N}}|z-a|\right)^{2}} \sum_{k=0}^{n} \frac{1}{k !(n-k) !} \\
& =\left(\min _{z \in \mathbb{N}}|z-a|\right)^{-2} \frac{a^{2} 2^{n}}{n !} .
\end{aligned}
$$

From

$$
\begin{aligned}
\int_{0}^{\infty} \frac{x^{m(a-1)+n-1} e^{-x}}{N_{u}(x)} \mathrm{d} x= & \int_{0}^{\infty} \frac{x^{m(a-1)+n-1} e^{-x}}{(c-\lambda \mu)^{2}} \mathrm{~d} x \\
& -\int_{0}^{\infty} \frac{\left(Z_{u}(x, 0,0,0)-(c-\lambda \mu)^{2}\right) x^{m(a-1)+n-1} e^{-x}}{(c-\lambda \mu)^{2} N_{u}(x)} \mathrm{d} x \\
= & \frac{\Gamma(m(a-1)+n))}{(c-\lambda \mu)^{2}}-\int_{0}^{\infty} \frac{Z_{u}(x, 1,0,0) x^{m(a-1)+n-1} e^{-x}}{(c-\lambda \mu)^{2} N_{u}(x)} \mathrm{d} x
\end{aligned}
$$

we get

$$
\begin{aligned}
\int_{0}^{\infty} & \frac{Z_{u}\left(x, n_{0}, n_{1}, n_{2}\right) x^{m(a-1)+n-1} e^{-x}}{N_{u}(x)} \mathrm{d} x \\
= & \frac{\left.c_{0} \Gamma((m+2)(a-1)+n)\right) I_{\left\{n_{2}=0\right\}}}{(c-\lambda \mu)^{2}} u^{-2(a-1)} \\
& +\frac{\left.d_{n_{1}} \Gamma\left((m+1)(a-1)+n+n_{1}\right)\right)}{(c-\lambda \mu)^{2}} u^{-(a-1)-n_{1}} \\
& +\frac{\left.e_{n_{0}} \Gamma\left(m(a-1)+n+n_{0}\right)\right)}{(c-\lambda \mu)^{2}} u^{-n_{0}} \\
& -I_{\left\{n_{2}=0\right\}} c_{0} u^{-2(a-1)} \int_{0}^{\infty} \frac{Z_{u}(x, 1,0,0) x^{(m+2)(a-1)+n-1} e^{-x}}{(c-\lambda \mu)^{2} N_{u}(x)} \mathrm{d} x \\
& -d_{n_{1}} u^{-(a-1)-n_{1}} \int_{0}^{\infty} \frac{Z_{u}(x, 1,0,0) x^{(m+1)(a-1)+n+n_{1}-1} e^{-x}}{(c-\lambda \mu)^{2} N_{u}(x)} \mathrm{d} x \\
& -e_{n_{0}} u^{-n_{0}} \int_{0}^{\infty} \frac{Z_{u}(x, 1,0,0) x^{m(a-1)+n+n_{0}-1} e^{-x}}{(c-\lambda \mu)^{2} N_{u}(x)} \mathrm{d} x \\
& +\int_{0}^{\infty} \frac{Z_{u}\left(x, n_{0}+1, n_{1}+1, n_{2}+1\right) x^{m(a-1)+n-1} e^{-x}}{N_{u}(x)} \mathrm{d} x .
\end{aligned}
$$


The terms with integrals depending on $u$ are of higher order than at least one of the other terms and these integrals are of the same type as the starting integral; hence one can iteratively get the complete asymptotic expansion of the first integral.

Since

$$
\psi(u) \approx \frac{\rho}{1-\rho} \bar{F}_{I}(u) \frac{(c-\lambda \mu)^{2}}{\Gamma(a-1)} \int_{0}^{\infty} \frac{x^{\alpha-2} e^{-x}}{N_{u}(x)} \mathrm{d} x,
$$

one can in this way obtain the complete asymptotic expansion of $\psi(s)$ in terms of $\bar{F}(s)^{\alpha_{i}}$, $\alpha_{i} \in \mathbb{R}$.

As a concrete example, we derive the asymptotic expansion for $\psi(u)$ up to the order of $f(u)=a u^{-a-1}$ for $a>2$. We get

$$
\begin{aligned}
\psi(u) \approx & \frac{\rho}{1-\rho} \bar{F}_{I}(u) \frac{(c-\lambda \mu)^{2}}{\Gamma(a-1)}\left(\frac{\Gamma(a-1)}{(c-\lambda \mu)^{2}}-\int_{0}^{\infty} \frac{Z_{u}(x, 1,0,0) x^{a-2} e^{-x}}{(c-\lambda \mu)^{2} N_{u}(x)} \mathrm{d} x\right) \\
= & \frac{\rho}{1-\rho} \bar{F}_{I}(u)-\frac{\rho}{1-\rho} \frac{\bar{F}_{I}(u)}{\Gamma(a-1)}\left(\frac{1}{u} \frac{e_{1} \Gamma(a)}{(c-\lambda \mu)^{2}}\right. \\
& \left.-\frac{e_{1}}{u} \int_{0}^{\infty} \frac{Z_{u}(x, 1,0,0) x^{a-1} e^{-x}}{(c-\lambda \mu)^{2} N_{u}(x)} \mathrm{d} x+\int_{0}^{\infty} \frac{Z_{u}(x, 2,0,0) x^{a-2} e^{-x}}{N_{u}(x)} \mathrm{d} x\right) \\
= & \frac{\rho}{1-\rho} \bar{F}_{I}(u)-e_{1} \frac{\rho}{1-\rho} \frac{a-1}{(c-\lambda \mu)^{2}} \frac{\bar{F}_{I}(u)}{u} \\
& +\frac{\rho}{1-\rho} \frac{e_{1} \bar{F}_{I}(u)}{u \Gamma(a-1)(c-\lambda \mu)^{2}}\left(\frac{1}{u} \frac{e_{1} \Gamma(a+1)}{(c-\lambda \mu)^{2}}\right. \\
& \left.-\frac{e_{1}}{u} \int_{0}^{\infty} \frac{Z_{u}(x, 1,0,0) x^{a} e^{-x}}{(c-\lambda \mu)^{2} N_{u}(x)} \mathrm{d} x+\int_{0}^{\infty} \frac{Z_{u}(x, 2,0,0) x^{a-1} e^{-x}}{N_{u}(x)} \mathrm{d} x\right) \\
& -\frac{\rho}{1-\rho} \frac{\bar{F}_{I}(u)}{\Gamma(a-1)}\left(\frac{1}{u^{2}} \frac{e_{2} \Gamma(a+1)}{(c-\lambda \mu)^{2}}\right. \\
& \left.-\frac{e_{2}}{u^{2}} \int_{0}^{\infty} \frac{Z_{u}(x, 1,0,0) x^{a} e^{-x}}{(c-\lambda \mu)^{2} N_{u}(x)} \mathrm{d} x+\int_{0}^{\infty} \frac{Z_{u}(x, 3,0,0) x^{a-2} e^{-x}}{N_{u}(x)} \mathrm{d} x\right) .
\end{aligned}
$$

For $a>3$ we get with

$$
\bar{F}_{I}(x)=\frac{1}{a} x^{-a+1}, \quad f_{I}(x)=\frac{a-1}{a} x^{-a} \quad \text { and } \quad f_{I}^{\prime}(x)=(1-a) x^{-a-1},
$$

so that

$$
\begin{aligned}
& \psi(u)=\frac{\rho}{1-\rho} \bar{F}_{I}(u)-\frac{\rho}{1-\rho} \frac{e_{1}}{(c-\lambda \mu)^{2}} f_{I}(u) \\
&+\left(\frac{\rho}{1-\rho} \frac{e_{1}^{2}}{(c-\lambda \mu)^{4}}-\frac{\rho}{1-\rho} \frac{e_{2}}{(c-\lambda \mu)^{2}}\right) f_{I}^{\prime}(u)+\mathcal{O}\left(u^{-\min \{a+2,2 a-2\}}\right) .
\end{aligned}
$$


From

$$
\begin{aligned}
& a_{0}=\frac{a}{1-a}=-\mu, \quad a_{1}=\frac{a}{2(1-a)}=-\frac{\mu_{2}}{2}, \quad a_{2}=\frac{a}{6(3-a)}=-\frac{\mu_{3}}{6} \quad \text { and } \\
& b_{1}=2 a_{0} a_{1}=\mu \mu_{2}, \quad b_{2}=2 a_{0} a_{2}+a_{1}^{2}=\frac{\mu \mu_{3}}{3}+\frac{\mu_{2}^{2}}{4},
\end{aligned}
$$

and

$$
e_{1}=\lambda^{2} b_{1}+2 \lambda c a_{1}=-\lambda \mu_{2}(c-\lambda \mu) \quad \text { and } \quad e_{2}=\lambda^{2} b_{2}+2 \lambda c a_{2}=\frac{\lambda^{2} \mu_{2}^{2}}{4}-\frac{\lambda \mu_{3}}{c-\lambda \mu},
$$

it follows that

$$
-\frac{\rho}{1-\rho} \frac{e_{1}}{(c-\lambda \mu)^{2}} f_{I}(u)=\frac{\rho}{1-\rho} \frac{\lambda \mu}{c-\lambda \mu} \frac{\mu_{2}}{\mu} f_{I}(u)=\frac{\rho^{2}}{(1-\rho)^{2}} \frac{\mu_{2}}{\mu} f_{I}(u)=\frac{\rho^{2}}{(1-\rho)^{2}} \frac{\mu_{2}}{\mu^{2}} \bar{F}(u),
$$

and

$$
\begin{aligned}
& \left(\frac{\rho}{1-\rho} \frac{e_{1}^{2}}{(c-\lambda \mu)^{4}}-\frac{\rho}{1-\rho} \frac{e_{2}}{(c-\lambda \mu)^{2}}\right) f_{I}^{\prime}(u) \\
& =\left(-\frac{\rho^{3}}{(1-\rho)^{3}} \frac{\mu_{2}^{2}}{\mu^{2}}+\frac{\rho}{1-\rho} \frac{\lambda^{2} \mu^{2}}{(c-\lambda \mu)^{2}} \frac{\mu_{2}^{2}}{4 \mu^{2}}-\frac{\rho}{1-\rho} \frac{\lambda \mu}{(c-\lambda \mu)^{2}} \frac{\mu_{3}}{3 \mu}\right) f_{I}^{\prime}(u) \\
& =\left(\frac{\rho^{3}}{(1-\rho)^{3}} \frac{3 \mu_{2}^{2}}{4 \mu^{3}}+\frac{\rho^{2}}{(1-\rho)^{2}} \frac{\mu_{3}}{3 \mu^{2}}\right) f(u) .
\end{aligned}
$$

which is in accordance with (18).

For $a<3$, clearly $2(a-1)<a+1$, so that we have to add further terms. We get

$$
\begin{aligned}
\int_{0}^{\infty} \frac{Z_{u}(x, 3,0,0) x^{a-2} e^{-x}}{N_{u}(x)} \mathrm{d} x= & \frac{d_{0} \Gamma(2(a-1))}{(c-\lambda \mu)^{2}} u^{-(a-1)} \\
& -d_{0} u^{-(a-1)} \int_{0}^{\infty} \frac{Z_{u}(x, 1,0,0) x^{2(a-1)-1} e^{-x}}{(c-\lambda \mu)^{2} N_{u}(x)} \mathrm{d} x \\
& +\int_{0}^{\infty} \frac{Z_{u}(x, 3,1,0) x^{a-2} e^{-x}}{N_{u}(x)} \mathrm{d} x \\
= & \frac{d_{0} \Gamma(2(a-1))}{(c-\lambda \mu)^{2}} u^{-(a-1)}+\mathcal{O}\left(\left(u^{-\min \{a, 2 a-2,3\}}\right)\right) .
\end{aligned}
$$

By defining $\mu_{3}=a /(a-3)$, even if the third moment does not exist, we obtain for every noninteger $a>2$ the expansion (19). For $a<2$, one has to insert even further terms to get an asymptotic expansion with the desired accuracy.

\section{Integral representations for compound sums}

In this section we are concerned with the collective risk model, i.e. we assume that $S_{N}=X_{1}+\ldots+X_{N}$, where the $X_{i}$ are iid Pareto and $N$ is an integer-valued random variable independent of the $X_{i}$. We are interested in

$$
\bar{G}(u)=\mathbb{P}\left(S_{N}>u\right) .
$$


If we denote with $Q_{N}(z)=\mathbb{E}\left[z^{N}\right]$, then

$$
\hat{L}_{\bar{G}}(s)=\frac{1}{s}-\frac{1}{s} Q_{N}\left(\hat{L}_{f}(s)\right),
$$

and for an $s_{0}>0$ we get

$$
\bar{G}(u)=1-\frac{1}{2 \pi \iota} \int_{s_{0}-\iota \infty}^{s_{0}+\iota \infty} e^{u s} \frac{1}{s} Q_{N}\left(\hat{L}_{f}(s)\right) \mathrm{d} s .
$$

Theorem 4.1. Let $X_{1}, X_{2}, \ldots$ be iid Pareto random variables with density $f(x)=a x^{-a-1}$ and $N$ be an integer-valued random variable independent of $X_{i}$ such that there exists an $\epsilon>0$ with

$$
\mathbb{E}\left[(1+\epsilon)^{N}\right]=\sum_{n=0}^{\infty} p_{n}(1+\epsilon)^{n}<\infty,
$$

where $p_{n}=\mathbb{P}(N=n)$. Then there exists a $t_{0}>0$ with

$$
\mathbb{P}\left(S_{N}>u\right)=\bar{G}(u) \approx-\frac{1}{\pi} \int_{0}^{t_{0}} \frac{1}{x} e^{-u x} \operatorname{Im}\left(Q_{N}\left(\hat{L}_{f}(-x)\right)\right) \mathrm{d} x .
$$

If further $Q_{N}\left(\hat{L}_{f}(-x)\right)$ exists for all $x>0$ and for a $\delta_{0}>0$

$$
\int_{0}^{\infty}\left|e^{-\delta_{0} x} \frac{1}{x} \operatorname{Im}\left(Q_{N}\left(\hat{L}_{f}(-x)\right)\right)\right|<\infty
$$

then

$$
\mathbb{P}\left(S_{N}>u\right)=\bar{G}(u) \approx-\frac{1}{\pi} \int_{0}^{\infty} \frac{1}{x} e^{-u x} \operatorname{Im}\left(Q_{N}\left(\hat{L}_{f}(-x)\right)\right) \mathrm{d} x .
$$

Proof. We would like to apply Lemma 2.9 with

$$
g(s)=Q_{N}\left(\hat{L}_{f}(s)\right) .
$$

At first we have to show that $g(s)$ is holomorphic on $\mathbb{D} \cap\left\{s: \operatorname{Re}(s)>-t_{0}\right\}$. Since $\sum_{n=0}^{\infty} p_{n}(1+\epsilon)^{n}<\infty$, it is enough to show that there exists a $t_{0}$ such that $\left|\hat{L}_{f}(s)\right|<$ $1+\epsilon / 2$ on $\left\{s: \operatorname{Re}(s)>-t_{0}\right\}$. Since $\left|\hat{L}_{f}(s)\right| \leq 1$ for $\operatorname{Re}(s) \geq 0, \lim _{|s| \rightarrow \infty}\left|\hat{L}_{f}(s)\right|=0$ on $\left\{s: \operatorname{Re}(s)>-t_{1}\right\}$ for every $t_{1}>0$ and $\left|\hat{L}_{f}(s)\right|$ is continuous on $\left\{s: \operatorname{Re}(s)>-t_{1}\right\}$ for every $t_{1}>0$, it follows by continuity arguments that there exists a $t_{0}>0$ such that $\left\{s: \operatorname{Re}(s)>-t_{1}\right\}$ for every $t_{1}>0$.

Clearly we have $g(\bar{s})=\overline{g(s)}$. From $\lim _{s \rightarrow 0} \hat{L}_{f}(s)=1$ and $\mathbb{E}\left[(1+\epsilon)^{N}\right]<\infty$ it follows that $\lim _{s \rightarrow 0} g(s)=\lim _{s \rightarrow 0} \mathbb{E}\left[\left(\hat{L}_{f}(s)\right)^{N}\right]=1$. Denote by $m \geq 1$ the smallest positive integer with $p_{m} \neq 0$, then uniformly for bounded sets of $\alpha>-t_{0}$ :

$$
\left|p_{0}-g(\alpha+\iota \beta)\right| \leq\left|\hat{L}_{f}(\alpha+\iota \beta)\right|^{m} \sum_{n=0}^{\infty} p_{m+n}\left|\hat{L}_{f}(\alpha+\iota \beta)\right|^{n}=\mathcal{O}\left(\frac{1}{\beta^{m}}\right) .
$$




\subsection{The compound Poisson model}

Assume that $N \sim \operatorname{Poisson}(\lambda)$, i.e. $\mathbb{P}(N=n)=e^{-\lambda} \frac{\lambda^{n}}{n !}$. Note that

$$
\hat{L}_{\bar{G}}(s)=\frac{1}{s}-\frac{1}{s} e^{-\lambda\left(1-\hat{L}_{f}(s)\right)}=\frac{1}{s}-\frac{1}{s} e^{-\lambda\left(1-a s^{a} \Gamma(-a, s)\right)},
$$

is holomorphic on $\mathbb{D}$. From Theorem 4.1 we get

$$
\bar{G}(u) \approx \frac{1}{\pi} \int_{0}^{\infty} \frac{1}{x} e^{-u x-\lambda \operatorname{Re}\left(1-a(-x)^{a} \Gamma(-a,-x)\right)} \sin \left(\frac{\lambda x^{a} \pi}{\Gamma(a)}\right) \mathrm{d} x .
$$

Remark 4.1. The integral in (21) is oscillating, but it is at least absolutely integrable, which is not the case for the integral in (20). Further note that

$$
\operatorname{Re}\left(1-a(-x)^{a} \Gamma(-a,-x)\right) \sim \frac{a}{x} e^{x},
$$

so that the integrand decays very quickly for large $x$, which makes a numerical evaluation of the integral quite feasible.

\subsection{The compound negative binomial model}

For the negative binomial distribution given by

$$
\mathbb{P}(N=n)=\left(\begin{array}{c}
r+n-1 \\
n
\end{array}\right) \rho^{r}(1-\rho)^{n}, \quad 0<\rho<1, r>0
$$

and

$$
Q_{N}(z)=\left(\frac{\rho}{1-(1-\rho) z}\right)^{r}
$$

Theorem 4.1 gives

$$
\mathbb{P}\left(S_{N}>u\right)=\bar{G}(u) \approx-\frac{1}{\pi} \int_{0}^{\infty} \frac{1}{x} e^{-u x} \operatorname{Im}\left(\left(\frac{\rho}{1-(1-\rho) a(-x)^{a} \Gamma(-a,-x)}\right)^{r}\right) \mathrm{d} x .
$$

\section{$5 \quad$ Numerical examples}

We now provide numerical illustrations for the performance of the derived approximations to $\psi(u)$ and $\bar{G}(u)$, respectively. We will compare the approximations with the first order asymptotic approximations given by $\rho /(1-\rho) \bar{F}_{I}(u)$ and $\mathbb{E}[N] \bar{F}(u)$, respectively. We also include a second order asymptotic approximation (containing the next significant asymptotic term). For a compound sum with $\mathbb{E}[X]<\infty$ and regularly varying density $f(x)$, this extra term is $2 \mathbb{E}\left[\left(\begin{array}{c}N \\ 2\end{array}\right)\right] \mathbb{E}[X] f(u)$. When $f(x)$ is regularly varying with index $\alpha=a+1=2$, then the next term is $2 \mathbb{E}\left[\left(\begin{array}{c}N \\ 2\end{array}\right)\right] f(u) \int_{0}^{u} \bar{F}(x) \mathrm{d} x$. In principle, there is also a next asymptotic term when $f(x)$ is regularly varying with index $\alpha=a+1<2$, but in the case $a=1 / 2$ (which is the case in our examples) it turns out that this term is 0 and hence we omit the second order approximation for the compound sums in this case (see e.g. [20] and [2] for further details on these higher order expansions). In the 
case of the ruin probability, we use the Pollaczek-Khintchine formula to rewrite the ruin probability as a compound sum, for the case $a=1.5$ we use the next term in the expansion given in Section 3. Furthermore we use a Monte Carlo estimate with 100000 iterations. We use the estimator $N \bar{F}\left(\max \left(M_{N-1},\left(u-S_{N-1}\right)\right)\right)$, where $M_{N-1}=\max \left(X_{1}, \ldots, X_{N-1}\right)$ of [4]. This estimator is proven to be asymptotically efficient for compound sums. To provide upper and lower bounds for the actual values of $\psi(u)$ and $\bar{G}(u)$, respectively, we use Panjer recursions with stepsize 0.01 for the ruin probabilities and 0.1 for the compound sums (cf. [11, Chapter 6.6]). To compare the efficiency of the approximations, the needed computation times of the Monte Carlo estimate, Panjer bounds and the integral approximations are provided (Panjer recursions were implemented in $C++$, whereas all other calculations where done using Mathematica 6.0). We will see that the integral approximations provide excellent results whereas the common asymptotic approximations can be far off the correct values.

\subsection{Approximation of the ruin probability}

For the risk process we choose the parameters $a=1.5, \lambda=1, c=3.5$ or $a=1.5, \lambda=1$, $c=2.5$ or $a=2.5, \lambda=1, c=2$, which corresponds to $\rho=0.857, \rho=0.8$ or $\rho=0.833$, respectively. For each of these examples, the Panjer recursion for the evaluation of upper and lower bounds needs 390 seconds. A Monte Carlo simulation of sufficient accuracy takes between 8 and 14 seconds and the numerical evaluation of the integral (5) takes approximately 0.04 seconds. The estimates are given in Table 1, 2 or 3 respectively.

\begin{tabular}{||c|c|c|c|c|c|c||}
\hline $\mathrm{u}$ & upper bound & lower bound & integral & first order & second order & $\mathrm{MC}$ \\
\hline 16 & 0.56549 & 0.56513 & 0.56535 & 1 & 1.03406 & 0.56447 \\
30 & 0.48582 & 0.48557 & 0.48572 & 0.73030 & 0.74356 & 0.48659 \\
50 & 0.41931 & 0.41913 & 0.41924 & 0.56569 & 0.57185 & 0.41893 \\
100 & 0.33189 & 0.33179 & 0.33185 & 0.4 & 0.40218 & 0.33126 \\
500 & 0.17017 & 0.17016 & 0.17017 & 0.17889 & 0.17908 & 0.16955 \\
1000 & 0.12320 & 0.12319 & 0.12320 & 0.12649 & 0.12656 & 0.12312 \\
\hline
\end{tabular}

Table 1: Numerical estimates of ruin probabilities with different methods with $a=1.5$, $\lambda=1, c=3.5$ and $\rho=0.857$

\begin{tabular}{||c|c|c|c|c|c|c||}
\hline $\mathrm{u}$ & upper bound & lower bound & integral & first order & second order & $\mathrm{MC}$ \\
\hline 16 & 0.20141 & 0.20058 & 0.20105 & 0.12500 & 0.25852 & 0.20069 \\
30 & 0.10591 & 0.10553 & 0.10574 & 0.6667 & 0.11023 & 0.10571 \\
50 & 0.05795 & 0.05780 & 0.05788 & 0.04 & 0.05732 & 0.05792 \\
100 & 0.02507 & 0.02503 & 0.02505 & 0.02 & 0.02488 & 0.02496 \\
500 & 0.00423 & 0.00423 & 0.00423 & 0.00400 & 0.00425 & 0.00423 \\
1000 & 0.00206 & 0.00206 & 0.00206 & 0.00200 & 0.00207 & 0.00206 \\
\hline
\end{tabular}

Table 2: Numerical estimates of ruin probabilities with different methods with $a=2$, $\lambda=1, c=2.5$ and $\rho=0.8$ 


\begin{tabular}{|c|c|c|c|c|c|c||}
\hline $\mathrm{u}$ & upper bound & lower bound & integral & first order & second order & $\mathrm{MC}$ \\
\hline 16 & 0.11888 & 0.11757 & 0.11828 & 0.3125 & 0.7520 & 0.12006 \\
30 & 0.03581 & 0.03539 & 0.03561 & 0.01217 & 0.02130 & 0.03529 \\
50 & 0.01109 & 0.01100 & 0.01 .105 & 0.00566 & 0.00820 & 0.01095 \\
100 & 0.00264 & 0.00263 & 0.00264 & 0.00200 & 0.00245 & 0.00262 \\
500 & 0.00019 & 0.00019 & 0.00019 & 0.00018 & 0.00019 & 0.00019 \\
1000 & 0.00006 & 0.00006 & 0.00006 & 0.00006 & 0.00006 & 0.00007 \\
\hline
\end{tabular}

Table 3: Numerical estimates of ruin probabilities with different methods with $a=2.5$, $\lambda=1, c=2$, and $\rho=0.833$

\subsection{Compound Poisson}

Let us now consider an example for the calculation of the tail of a compound Poisson sum of Pareto(0.5), Pareto(1) and Pareto(1.5) claims with $\lambda=2$. In this case the Panjer recursion takes 4 seconds to evaluate upper and lower bounds. The Monte Carlo simulation takes 14 seconds and the evaluation of each integral takes between 0.02 and 0.06 seconds. The estimates are given in Tables 4, 5 and 6 :

\begin{tabular}{||c|c|c|c|c|c|}
\hline $\mathrm{u}$ & upper bound & lower bound & integral & first order & $\mathrm{MC}$ \\
\hline 16 & 0.44592 & 0.44318 & 0.44508 & 0.50000 & 0.44257 \\
30 & 0.34268 & 0.34132 & 0.34224 & 0.36515 & 0.34192 \\
50 & 0.27207 & 0.27136 & 0.27184 & 0.28284 & 0.27026 \\
100 & 0.19610 & 0.19583 & 0.19601 & 0.20000 & 0.19565 \\
500 & 0.08909 & 0.08906 & 0.08908 & 0.08944 & 0.08896 \\
1000 & 0.06312 & 0.06311 & 0.06312 & 0.06325 & 0.06309 \\
\hline
\end{tabular}

Table 4: Numerical estimates of tail probabilities for a compound Poisson sum with $a=0.5$ and $\lambda=2$ with different methods

\begin{tabular}{||c|c|c|c|c|c|c||}
\hline $\mathrm{u}$ & upper bound & lower bound & integral & first order & second order & $\mathrm{MC}$ \\
\hline 16 & 0.16942 & 0.16573 & 0.16811 & 0.12500 & 0.18395 & 0.16805 \\
30 & 0.08336 & 0.08233 & 0.08299 & 0.06667 & 0.08623 & 0.08301 \\
50 & 0.04689 & 0.04655 & 0.04677 & 0.04000 & 0.04786 & 0.04675 \\
100 & 0.02198 & 0.02190 & 0.02195 & 0.02000 & 0.02224 & 0.02192 \\
500 & 0.00410 & 0.00410 & 0.00410 & 0.00400 & 0.00412 & 0.00409 \\
1000 & 0.00203 & 0.00203 & 0.00203 & 0.00200 & 0.00203 & 0.00203 \\
\hline
\end{tabular}

Table 5: Numerical estimates of tail probabilities for a compound Poisson sum with $a=1$ and $\lambda=2$ with different methods 


\begin{tabular}{||c|c|c|c|c|c|c||}
\hline $\mathrm{u}$ & upper bound & lower bound & integral & first order & second order & $\mathrm{MC}$ \\
\hline 16 & 0.05487 & 0.05248 & 0.05396 & 0.03125 & 0.04883 & 0.05400 \\
30 & 0.01670 & 0.01635 & 0.01657 & 0.01217 & 0.01582 & 0.01646 \\
50 & 0.00683 & 0.00676 & 0.00680 & 0.00566 & 0.00668 & 0.00682 \\
100 & 0.00220 & 0.00218 & 0.00219 & 0.00200 & 0.00218 & 0.00219 \\
500 & 0.00018 & 0.00018 & 0.00018 & 0.00018 & 0.00018 & 0.00018 \\
1000 & 0.00006 & 0.00006 & 0.00006 & 0.00006 & 0.00006 & 0.00006 \\
\hline
\end{tabular}

Table 6: Numerical estimates of tail probabilities for a compound Poisson sum with $a=$ $1.5, \lambda=2$ and $\mathbb{E}\left[S_{N}\right]=6$ with different methods

\subsection{Compound negative binomial}

Finally, we consider the determination of the tail of a compound negative binomial sum of Pareto(0.5), Pareto(1) and Pareto(1.5) claims with $\rho=0.5$ and $r=3$. The Panjer recursion takes 4 seconds to evaluate upper and lower bounds. A Monte Carlo simulation takes 17 seconds and the evaluation of the integral takes between 0.02 and 0.04 seconds. The estimates are given in Tables 7,8 and 9 .

\begin{tabular}{||c|c|c|c|c|c|}
\hline $\mathrm{u}$ & upper bound & lower bound & integral & first order & $\mathrm{MC}$ \\
\hline 16 & 0.55915 & 0.55611 & 0.55812 & 0.75 & 0.57117 \\
30 & 0.45624 & 0.45432 & 0.45554 & 0.54772 & 0.47091 \\
50 & 0.37635 & 0.37516 & 0.3759 & 0.42426 & 0.34788 \\
100 & 0.28116 & 0.28062 & 0.28095 & 0.30 & 0.29469 \\
500 & 0.1323 & 0.13224 & 0.13228 & 0.13416 & 0.13302 \\
1000 & 0.0942 & 0.09418 & 0.09419 & 0.09487 & 0.09775 \\
\hline
\end{tabular}

Table 7: Numerical estimates of tail probabilities for a compound negative binomial sum with $a=0.5, \rho=0.5$ and $r=3$ with different methods

\begin{tabular}{||c|c|c|c|c|c|c||}
\hline $\mathrm{u}$ & upper bound & lower bound & integral & first order & second order & $\mathrm{MC}$ \\
\hline 16 & 0.2881 & 0.28093 & 0.28525 & 0.1875 & 0.36434 & 0.28407 \\
30 & 0.15035 & 0.14733 & 0.14908 & 0.10 & 0.15868 & 0.1486 \\
50 & 0.08259 & 0.08147 & 0.08211 & 0.06 & 0.08358 & 0.0816 \\
100 & 0.03649 & 0.03625 & 0.03639 & 0.03 & 0.03673 & 0.03657 \\
500 & 0.00632 & 0.00631 & 0.00631 & 0.006 & 0.00635 & 0.00635 \\
1000 & 0.00309 & 0.00308 & 0.00309 & 0.003 & 0.00309 & 0.00308 \\
\hline
\end{tabular}

Table 8: Numerical estimates of tail probabilities for a compound negative binomial sum with $a=1, \rho=0.5$ and $r=3$ with different methods 


\begin{tabular}{||c|c|c|c|c|c|c||}
\hline $\mathrm{u}$ & upper bound & lower bound & integral & first order & second order & $\mathrm{MC}$ \\
\hline 16 & 0.13498 & 0.12677 & 0.13146 & 0.04688 & 0.09961 & 0.13212 \\
30 & 0.03825 & 0.03643 & 0.03744 & 0.01826 & 0.02921 & 0.03737 \\
50 & 0.01314 & 0.0128 & 0.01299 & 0.00849 & 0.01154 & 0.0129 \\
100 & 0.00367 & 0.00363 & 0.00365 & 0.003 & 0.00354 & 0.00364 \\
500 & 0.00028 & 0.00028 & 0.00028 & 0.00027 & 0.00028 & 0.00028 \\
1000 & 0.0001 & 0.0001 & 0.0001 & 0.00009 & 0.0001 & 0.0001 \\
\hline
\end{tabular}

Table 9: Numerical estimates of tail probabilities for a compound negative binomial sum with $a=1.5, \rho=0.5, r=3$ and $\mathbb{E}\left[S_{N}\right]=9$ with different methods

\section{References}

[1] M. Abramowitz and I. A. Stegun. Handbook of mathematical functions with formulas, graphs, and mathematical tables, volume 55 of National Bureau of Standards Applied Mathematics Series. Washington, D.C., 1964.

[2] H. Albrecher, C. Hipp, and D. Kortschak. Higher order expansions for compound distributions and ruin probabilities with subexponential claims. Scand. Actuar. J., 2009, To appaer.

[3] S. Asmussen. Ruin probabilities, volume 2 of Advanced Series on Statistical Science Es Applied Probability. World Scientific Publishing Co. Inc., River Edge, NJ, 2000.

[4] S. Asmussen and D. P. Kroese. Improved algorithms for rare event simulation with heavy tails. Adv. in Appl. Probab., 38(2):545-558, 2006.

[5] A. Baltrūnas. Second order behaviour of ruin probabilities. Scand. Actuar. J., (2):120$133,1999$.

[6] P. Barbe and W. P. McCormick. Asymptotic expansions for infinite weighted convolutions of heavy tail distributions and applications. American Mathematical Society, 2009.

[7] M. Blum. On the sums of independently distributed Pareto variates. SIAM J. Appl. Math., 19:191-198, 1970.

[8] L. Brennan, I. Reed, and W. Sollfrey. A comparison of average-likelihood and maximum-likelihood ratio tests for detecting radar targets of unknown Doppler frequency. IEEE Trans. Information Theory, 14(1):104-110, 1968.

[9] P. Embrechts and N. Veraverbeke. Estimates for the probability of ruin with special emphasis on the possibility of large claims. Insurance Math. Econom., 1(1):55-72, 1982.

[10] R. Grübel. On subordinated distributions and generalized renewal measures. Ann. Probab., 15(1):394-415, 1987. 
[11] S. A. Klugman, H. H. Panjer, and G. E. Willmot. Loss models. Wiley Series in Probability and Statistics: Applied Probability and Statistics. John Wiley \& Sons Inc., New York, 1998.

[12] F. W. J. Olver. Asymptotics and special functions. AKP Classics. A K Peters Ltd., Wellesley, MA, 1997.

[13] E. Omey and E. Willekens. Second-order behaviour of distributions subordinate to a distribution with finite mean. Comm. Statist. Stochastic Models, 3(3):311-342, 1987.

[14] C. M. Ramsay. A solution to the ruin problem for Pareto distributions. Insurance Math. Econom., 33(1):109-116, 2003.

[15] C. M. Ramsay. Exact waiting time and queue size distributions for equilibrium $M / G / 1$ queues with Pareto service. Queueing Syst., 57(4):147-155, 2007.

[16] C. M. Ramsay. The distribution of compound sums of Pareto distributed losses. Scandinavian Actuarial Journal, (1):27-37, 2009.

[17] T. Rolski, H. Schmidli, V. Schmidt, and J. Teugels. Stochastic processes for insurance and finance. Wiley Series in Probability and Statistics. John Wiley \& Sons Ltd., Chichester, 1999.

[18] J. Teugels and N. Veraverbeke. Cramér-type estimates for the probability of ruin. Technical report, C.O.R.E. Discussion Paper No 7316, 1973.

[19] L. Wei and H. Yang. Explicit expressions for the ruin probabilities in Erlang risk processes with Pareto individual claim distributions. Acta Math. Appl. Sin. Engl. Ser., 20(3):495-506, 2004.

[20] E. Willekens. Asymptotic approximations of compound distributions and some applications. Bull. Soc. Math. Belg. Sér. B, 41(1):55-61, 1989.

[21] E. Willekens and J. L. Teugels. Asymptotic expansions for waiting time probabilities in an $M / G / 1$ queue with long-tailed service time. Queueing Systems Theory Appl., 10(4):295-311, 1992.

\section{A Proofs of the auxiliary lemmata}

Proof of Lemma 2.2. The lemma is a direct consequence of the asymptotic behaviour of the incomplete Gamma function (14).

Proof of Lemma 2.3. Clearly, $\cos \beta=0$ and $\sin \beta=1$, hence we have

$$
s^{-2} e^{-s}=\frac{2 \alpha \beta-\iota\left(\alpha^{2}-\beta^{2}\right)}{\left(\alpha^{2}+\beta^{2}\right)^{2}} e^{\alpha},
$$

and $\operatorname{Re}\left(s^{-2} e^{-s}\right)>0$. Using the asymptotic behaviour of the incomplete Gamma function (14) we get

$$
N(s)=c+a \lambda s^{-2} e^{-s}-a \lambda s^{-2} e^{-s}\left(1-\frac{\Gamma(-a, s)}{s^{-a-1} e^{-s}}\right)-\frac{\lambda}{s} .
$$


Now choose $k_{0}>0$ such that for $k>k_{0}(s=\alpha+\iota(2 k+1) \pi / 2)$

$$
\left|1-\frac{\Gamma(-a, s)}{s^{-a-1} e^{-s}}\right|<\frac{\epsilon}{2 c} \quad \text { and } \quad \frac{\lambda}{|s|}<\frac{\epsilon}{2} \text {. }
$$

This gives

$$
\begin{aligned}
|N(s)| \geq\left|c+a \lambda s^{-2} e^{-s}\right|-\left|a \lambda s^{-2} e^{-s}\right|\left|1-\frac{\Gamma(-a, s)}{s^{-a-1} e^{-s}}\right| & -\frac{\lambda}{|s|} \\
& \geq\left|c+a \lambda s^{-2} e^{-s}\right|\left(1-\frac{\epsilon}{2 c}\right)-\frac{\epsilon}{2} \geq c-\epsilon .
\end{aligned}
$$

Proof of Lemma 2.4. Choose $\beta_{0}>0$ such that for $\beta>\beta_{0}$ both $\lambda /|s| \leq \epsilon$,

$$
\left|1-\frac{\Gamma(-a, s)}{s^{-a-1} e^{-s}}\right| \leq \epsilon
$$

hold and further for a $\delta>0$ and for all $\beta_{1}>\beta_{0}$ and all $\alpha_{1}>\sqrt{\epsilon} \beta_{1}$ it is valid that $N\left(-\alpha_{1}+\iota \beta_{1}\right)>\delta$ (compare Lemma 2.2). Since $N(s)=0$ we get that $\alpha \leq \sqrt{\epsilon} \beta$ and

$$
|c|=\left|a \lambda s^{-2} e^{-s}-a \lambda s^{-2} e^{-s}\left(1-\frac{\Gamma(-a, s)}{s^{-a-1} e^{-s}}\right)-\frac{\lambda}{s}\right|,
$$

from which we can deduce

$$
\frac{c+\epsilon}{a \lambda(1-\epsilon)} \geq\left|s^{-2} e^{-s}\right| \geq \frac{c-\epsilon}{a \lambda(1+\epsilon)}
$$

Since

$$
\left|s^{-2} e^{-s}\right|=\frac{e^{\alpha}}{\alpha^{2}+\beta^{2}}
$$

we get

$$
\alpha \geq \log \left(\frac{c-\epsilon}{a \lambda(1+\epsilon)}\left(\alpha^{2}+\beta^{2}\right)\right) \geq \log \left(\frac{(c-\epsilon) \beta^{2}}{a \lambda(1+\epsilon)}\right)
$$

and

$$
\alpha \leq \log \left(\frac{c+\epsilon}{a \lambda(1-\epsilon)}\left(\alpha^{2}+\beta^{2}\right)\right) \leq \log \left(\frac{(1+\epsilon)(c+\epsilon) \beta^{2}}{a \lambda(1-\epsilon)}\right) .
$$

Proof of Lemma 2.5. At first choose an $\epsilon_{1}>0$ such that for all $x$ with $|1-\cos x|<\epsilon_{1}$ it follows that $|\sin x|<\epsilon$, note that this is possible since $\sin x$ and the inverse of $\cos x$ are continuous. In view of (22), choose $\beta_{1}>0$ such that for all $\beta>\beta_{1}$

$$
\left|a \lambda s^{-2} e^{-s}\left(1-\frac{\Gamma(-a, s)}{s^{-a-1} e^{-s}}\right)+\frac{\lambda}{s}\right|<\frac{c \epsilon_{1}}{2} .
$$


We have

$$
\begin{aligned}
N(s)= & c+a \lambda s^{-2} e^{-s}-a \lambda s^{-2} e^{-s}\left(1-\frac{\Gamma(-a, s)}{s^{-a-1} e^{-s}}\right)-\frac{\lambda}{s}, \\
\operatorname{Re}(N(s))= & c+\frac{a \lambda e^{\alpha}}{\alpha^{2}+\beta^{2}}\left(\frac{\alpha^{2}-\beta^{2}}{\alpha^{2}+\beta^{2}} \cos (\beta)+\frac{2 \alpha \beta}{\alpha^{2}+\beta^{2}} \sin (\beta)\right) \\
& -\operatorname{Re}\left(a \lambda s^{-2} e^{-s}\left(1-\frac{\Gamma(-a, s)}{s^{-a-1} e^{-s}}\right)+\frac{\lambda}{s}\right), \\
\operatorname{Im}(N(s))= & \frac{a \lambda e^{\alpha}}{\alpha^{2}+\beta^{2}}\left(\frac{2 \alpha \beta}{\alpha^{2}+\beta^{2}} \cos (\beta)-\frac{\alpha^{2}-\beta^{2}}{\alpha^{2}+\beta^{2}} \sin (\beta)\right) \\
& -\operatorname{Im}\left(a \lambda s^{-2} e^{-s}\left(1-\frac{\Gamma(-a, s)}{s^{-a-1} e^{-s}}\right)+\frac{\lambda}{s}\right),
\end{aligned}
$$

and

$$
\begin{aligned}
|\operatorname{Re}(N(s))| \geq|c-c \cos (\beta)|-\mid c & -\frac{a \lambda \beta^{2} e^{\alpha}}{\left(\alpha^{2}+\beta^{2}\right)^{2}}|| \cos (\beta) \mid \\
& -\left|\frac{a \lambda e^{\alpha}}{\alpha^{2}+\beta^{2}}\left(\frac{\alpha^{2}}{\alpha^{2}+\beta^{2}} \cos (\beta)+\frac{2 \alpha \beta}{\alpha^{2}+\beta^{2}} \sin (\beta)\right)\right|-\frac{c \epsilon_{1}}{2} .
\end{aligned}
$$

Since from Lemma 2.4 , it follows that

$$
\lim _{\beta \rightarrow \infty}\left|c-\frac{a \lambda \beta^{2} e^{\alpha}}{\left(\alpha^{2}+\beta^{2}\right)^{2}}\right||\cos (\beta)|+\lim _{\beta \rightarrow \infty}\left|\frac{a \lambda e^{\alpha}}{\alpha^{2}+\beta^{2}}\left(\frac{\alpha^{2}}{\alpha^{2}+\beta^{2}} \cos (\beta)+\frac{2 \alpha \beta}{\alpha^{2}+\beta^{2}} \sin (\beta)\right)\right|=0,
$$

there exists a $\beta_{0}$ such that for $\beta>\beta_{0}$

$$
0=|\operatorname{Re}(N(x))| \geq c|1-\cos (\beta)|-c \epsilon_{1}, \quad \text { i.e. } \quad|1-\cos (\beta)| \leq \epsilon_{1} .
$$

Proof of Lemma 2.6. Let $k$ be large. Note that all zeros of $N(s)$ are poles of order one of the function $h(s)=N^{\prime}(s) / N(s)$ and the corresponding residual is 1 . Hence the number of zeros is

$$
\frac{1}{2 \pi \iota} \int_{K_{1}\left(s_{k}\right)} h(t) \mathrm{d} t
$$

We have

$$
h(s)=\frac{a(a-1) \lambda s^{a-2} \Gamma(-a, s)+\left(1-a e^{-s}\right) \lambda s^{-2}}{c-\frac{\lambda}{s}\left(1-a s^{a} \Gamma(-a, s)\right)}=\frac{(a-1) s^{-1}+\frac{1}{a s^{a+1} \Gamma(-a, s)}-\frac{s^{-a-1} e^{-s}}{\Gamma(-a, s)}}{\frac{c}{a \lambda s^{a-1} \Gamma(-a, s)}-\frac{1}{a s^{a} \Gamma(-a, s)}+1}
$$

Note that $\left|s^{a} \Gamma(-a, s)\right| \rightarrow \infty$ as $|s| \rightarrow \infty$ and $s \in K_{1}\left(s_{k}\right)$ for a $k>0$. For $\phi \in[0,2 \pi)$ and $s_{\phi, k}=s_{k}+e^{\iota \phi}$ we have:

$$
\frac{c}{a \lambda s_{\phi, k}^{a-1} \Gamma\left(-a, s_{\phi, k}\right)}=\frac{c s_{\phi, k}^{2}}{a \lambda e^{-s_{\phi, k}}}-\frac{c s_{\phi, k}^{2}}{a \lambda e^{-s_{\phi, k}}}\left(1-\frac{s_{\phi, k}^{-a-1} e^{-s_{\phi, k}}}{\Gamma\left(-a, s_{\phi, k}\right)}\right),
$$


and for $\alpha_{k}=\log \left(c(2 k \pi)^{2} /(a \lambda)\right), \beta_{k}=2 k \pi$

$$
\begin{aligned}
\frac{c s_{\phi, k}^{2}}{a \lambda e^{-s_{\phi, k}}}=e^{\cos (\phi)} e^{\iota\left(\sin (\phi)+\beta_{k}\right)} & \\
& \times \frac{\left(\cos (\phi)-\alpha_{k}\right)^{2}-\left(\sin (\phi)+\beta_{k}\right)^{2}+2\left(\cos (\phi)-\alpha_{k}\right)\left(\sin (\phi)+\beta_{k}\right) \iota}{\beta_{k}^{2}},
\end{aligned}
$$

hence we get uniformly in $\phi$

$$
\lim _{k \rightarrow \infty} h\left(s_{\phi, k}\right)=-\frac{1}{1-e^{\cos (\phi)} e^{\iota(\sin (\phi))}},
$$

and it follows that

$$
\lim _{k \rightarrow \infty} \frac{1}{2 \pi \iota} \int_{K_{1}\left(s_{k}\right)} h(t) \mathrm{d} t=-\frac{1}{2 \pi} \int_{0}^{2 \pi} \frac{e^{\iota \phi}}{1-e^{\cos (\phi)} e^{\iota(\sin (\phi))}} \mathrm{d} x=1 .
$$

Note that $\frac{1}{2 \pi \iota} \int_{K_{1}\left(s_{k}\right)} h(t) \mathrm{d} t$ is an integer.

Proof of Lemma 2.7. Recall

$$
\begin{gathered}
N(s)=c-\frac{\lambda}{s}\left(1-a s^{a} \Gamma(-a, s)\right) . \\
N^{\prime}(s)=\frac{\lambda}{s^{2}}\left(1-a s^{a} \Gamma(-a, s)\right)-a \lambda s^{a-2} \Gamma(1-a, s) \\
=\frac{\lambda}{s^{2}}\left(1-a s^{a} \Gamma(-a, s)\right)+a^{2} \lambda s^{a-2} \Gamma(-a, s)-a \lambda s^{-2} e^{-s} \\
=a(a-1) \lambda s^{a-2} \Gamma(-a, s)+\left(1-a e^{-s}\right) \lambda s^{-2}, \\
N^{\prime \prime}(s)=(a-2) a(a-1) \lambda s^{a-3} \Gamma(-a, s) \\
-a(a-1) \lambda s^{-3} e^{-s}-2\left(1-a e^{-s}\right) \lambda s^{-3}+a \lambda s^{-2} e^{-s} \\
=a(a-1)(a-2) \lambda s^{a-3} \Gamma(-a, s)-\lambda s^{-3}\left(a(a-3) e^{-s}+2\right)+a \lambda s^{-2} e^{-s} .
\end{gathered}
$$

For the existence of an $s$ with $N(s)=N^{\prime}(s)=N^{\prime \prime}(s)=0$, the following three equations would have to be fulfilled at the same time:

$$
\begin{aligned}
\Gamma(-a, s) & =\frac{s^{-a}}{a}-\frac{c s^{-a+1}}{a \lambda}, \\
\Gamma(-a, s) & =\frac{\left(a e^{-s}-1\right)}{a(a-1) s^{a}}, \\
\Gamma(-a, s) & =\frac{\left(a(a-3) e^{-s}+2\right)-a s e^{-s}}{a(a-1)(a-2) s^{a}} .
\end{aligned}
$$

From (23) and (24) we get

$$
\begin{aligned}
\frac{s^{-a}}{a}-\frac{c s^{-a+1}}{a \lambda} & =\frac{\left(a e^{-s}-1\right)}{a(a-1) s^{a}}, \\
1-\frac{c s}{\lambda} & =\frac{a}{a-1} e^{-s}-\frac{1}{a-1}, \\
1-\frac{s}{\rho}-e^{-s} & =0 .
\end{aligned}
$$


From (24) and (25) we get

$$
\begin{aligned}
\frac{\left(a e^{-s}-1\right)}{a(a-1) s^{a}} & =\frac{\left(a(a-3) e^{-s}+2\right)-a s e^{-s}}{a(a-1)(a-2) s^{a}}, \\
a e^{-s}-1 & =\frac{a(a-3)}{a-2} e^{-s}+\frac{2}{a-2}-\frac{a s e^{-s}}{a-2}, \\
\frac{a}{a-2} e^{-s}-\frac{a}{a-2} & =\frac{a}{a-2} s e^{-s}, \\
e^{s} & =1-s .
\end{aligned}
$$

Substituting this into (26) one obtains

$$
\begin{gathered}
1-\frac{s}{\rho}-\frac{1}{1-s}=0, \\
1-s-\frac{\left(s-s^{2}\right)}{\rho}-1=0, \\
s(s-1-1 / \rho)=0, \\
s \in\left\{0, \frac{1+\rho}{\rho}\right\},
\end{gathered}
$$

but $N(1+1 / \rho) \neq 0$ and $N(0) \neq 0$.

For the second part of the assertion choose $\beta_{0}$ as in Lemma 2.4 and $s=-\alpha+\iota \beta$ with $N(s)=0$ to obtain

$$
\left|1-\frac{s}{\rho}-e^{-s}\right| \geq\left|e^{-s}\right|-\frac{|s|}{\rho}-1 \geq \frac{c-\epsilon}{a \lambda(1+\epsilon)} \beta^{2}-\frac{\sqrt{\alpha^{2}+\beta^{2}}}{\rho}-1,
$$

which tends to infinity as $\beta \rightarrow \infty$ and the result follows from (26).

Proof of Lemma 2.8. Choose $\beta_{1}>\max \left(\beta_{0}, M\right)$ where $\beta_{0}$ is according to Lemma 2.4 and $M$ is according to Lemma 2.7 such that for every $\beta>\beta_{1}$.

$$
0 \leq \frac{(c-\lambda \mu)\left(\frac{a \lambda(1+\epsilon)}{c-\epsilon}\right)^{k} \frac{\alpha^{2}+\beta^{2}}{\beta^{2}}}{a \lambda\left(\left(\frac{c-\epsilon}{a \lambda(1+\epsilon)}\right) \beta^{2}-\frac{\left(\alpha^{2}+\beta^{2}\right)^{1 / 2}}{\rho}-1\right)} \leq 1 .
$$

Since $N^{\prime}\left(s_{i}\right) \neq 0$ one has

$$
\left|\operatorname{Res}\left(1 / N\left(s_{i}\right)\right)\right|=\frac{1}{\left|N^{\prime}\left(s_{i}\right)\right|}=\frac{1}{\left|a(a-1) \lambda s_{i}{ }^{a-2} \Gamma\left(-a, s_{i}\right)+\left(1-a e^{-s_{i}}\right) \lambda s_{i}{ }^{-2}\right|} .
$$

From (23) it follows that

$$
\begin{aligned}
\left|N^{\prime}\left(s_{i}\right)\right| & =\left|a(a-1) \lambda s_{i}{ }^{a-2}\left(\frac{s_{i}{ }^{-a}}{a}-\frac{c s_{i}{ }^{-a+1}}{a \lambda}\right)+\left(1-a e^{-s_{i}}\right) \lambda s_{i}{ }^{-2}\right| \\
& =\left|\frac{a \lambda}{s_{i}{ }^{2}}\left(1-\frac{s_{i}}{\rho}-e^{-s_{i}}\right)\right| \geq \frac{a \lambda\left(e^{\alpha}-\left|s_{i}\right| / \rho-1\right)}{\left|s_{i}\right|^{2}} \\
& \geq \frac{a \lambda\left(\frac{c-\epsilon}{a \lambda(1+\epsilon)} \beta^{2}-\frac{\left(\alpha^{2}+\beta^{2}\right)^{1 / 2}}{\rho}-1\right)}{\alpha^{2}+\beta^{2}}
\end{aligned}
$$


so that the assertion is implied by

$$
\left|e^{u s_{i}}\right|=e^{-(u-k) \alpha} e^{-k \alpha} \leq e^{-(u-k) \alpha}\left(\frac{a \lambda(1+\epsilon)}{c-\epsilon}\right)^{k} \beta^{-2 k} .
$$

